TRANSACTIONS OF THE

AMERICAN MATHEMATICAL SOCIETY

Volume 364, Number 11, November 2012, Pages 5881-5911

S 0002-9947(2012)05553-X

Article electronically published on May 21, 2012

\title{
CYCLIC OPERAD FORMALITY FOR COMPACTIFIED MODULI SPACES OF GENUS ZERO SURFACES
}

\author{
JEFFREY GIANSIRACUSA AND PAOLO SALVATORE
}

\begin{abstract}
The framed little 2-discs operad is homotopy equivalent to the Kimura-Stasheff-Voronov cyclic operad of moduli spaces of genus zero stable curves with tangent rays at the marked points and nodes. We show that this cyclic operad is formal, meaning that its chains and its homology (the Batalin-Vilkovisky operad) are quasi-isomorphic cyclic operads. To prove this we introduce a new complex of graphs in which the differential is a combination of edge deletion and contraction, and we show that this complex resolves BV as a cyclic operad.
\end{abstract}

\section{INTRODUCTION}

We begin by recalling two closely related operads. The first is the little 2-discs operad of Boardman and Vogt, denoted $D_{2}$. The arity $n$ space, $D_{2}(n)$, is the space of embeddings of the disjoint union of $n$ discs into a standard disc, where each disc is embedded by a map which is a translation composed with a dilation. At the level of spaces, group complete algebras over this operad are 2-fold loop spaces, and at the level of homology an algebra over $H_{*}\left(D_{2}\right)$ is precisely a Gerstenhaber algebra.

The second operad is a variant of the little 2-discs; it is the framed little 2-discs operad, denoted $f D_{2}$, first introduced by Getzler [5]. Here each little disc is allowed to be embedded by a composition of a rotation in addition to a dilation and translation, so in particular, $f D_{2}(n)=D_{2}(n) \times\left(S^{1}\right)^{n}$. Getzler observed that algebras over the homology operad $H_{*}\left(f D_{2}\right)$ are precisely Batalin-Vilkovisky algebras, and at the space level the second author and Wahl [20] proved that a group complete algebra over $f D_{2}$ is a 2-fold loop space on a based space with a circle action.

These operads each have higher-dimensional analogues: for any $k>2$ there is the little $k$-discs operad $D_{k}$ and the framed little $k$-discs operad $f D_{k}$. They are defined in exactly the same way using $k$-discs in place of 2 -discs.

In this paper we shall be concerned with the operad formality of $f D_{2}$. We recall first the related notion of formality of a space.

1.1. Formality of spaces. A space is said to be formal if Sullivan's algebra of piecewise polynomial rational differential forms is quasi-isomorphic to the rational cohomology algebra (with zero differential) in the category of commutative DGAs. On a smooth manifold and over $\mathbb{R}$ or $\mathbb{C}$ one can instead use the de Rham algebra of

Received by the editors September 10, 2010 and, in revised form, January 25, 2011.

2010 Mathematics Subject Classification. Primary 18D50; Secondary 55P48, 14H15, 81Q30, $81 \mathrm{~T} 45$.

Key words and phrases. Cyclic operad, framed little discs, moduli of curves, operad formality, graph complex.

(C) 2012 American Mathematical Society Reverts to public domain 28 years from publication 
smooth real or complex-valued differential forms. The equivalence of commutative DGA formality over fields of characteristic zero follows from the obstruction theory of $[10$.

The individual spaces of the operads $D_{k}(n)$ and $f D_{k}(n)$ are formal. Arnold proved this for $D_{2}(n)$ (over $\mathbb{C}$ ) [1. Much later, Kontsevich proved the formality of the spaces $D_{k}(n)$ for $k \geq 2$ (over $\mathbb{R}$ ) in the course of studying the formality of $D_{k}$ as an operad [16, 15] as explained below. Formality of the spaces $f D_{k}(n)$ follows immediately since Lie groups are formal and products of formal spaces are formal.

1.2. Formality of operads. An operad $P$ in topological spaces is said to be formal over a field $k$ if, in the category of operads in chain complexes over $k$, the operad $C_{*}^{\text {sing }}(P ; k)$ of singular chains is quasi-isomorphic to the homology operad $H_{*}(P ; k)$ (equipped with the zero differential). In general, formality of an operad is independent of formality of the individual spaces. For operads without nullary or unary operations, the paper [9] proves a descent theorem, which shows that the operad formality property is independent of the ground field in characteristic zero; it is not yet known if this remains true in the presence of nontrivial nullary or unary operations. In applications of operad formality these operations are important, so we shall take care to distinguish over which field formality takes place.

Tamarkin [24] first proved that the operad $D_{2}$ is formal over $\mathbb{Q}$. His proof is algebraic in nature, using braid groups and Drinfeld associators. Kontsevich [16, 15] then sketched a different proof of this formality theorem over $\mathbb{R}$, which generalizes to show formality of $D_{k}$ for all $k \geq 2$ and has the additional advantage of simultaneously proving the formality as spaces of the individual spaces of these operads. Roughly speaking, Kontsevich works dually, constructing a cooperad of cochain complexes of graphs which maps to both the cochains and the cohomology of $D_{k}$ by quasi-isomorphisms. The full details of Kontsevich's proof have been explained nicely by Lambrechts and Volic [17]. Recently Severa and Willwacher [22] have shown that, for $D_{2}$, the operad formality quasi-isomorphisms of Kontsevich and Tamarkin are homotopic for a particular choice of Drinfeld associator.

Note that Kontsevich's method does not easily imply formality of the chains on $D_{k}$ in the category of DG-Hopf operads, as discussed in the introduction and section 3 of [17, because the integration map from forms to cochains is not multiplicative.

Turning now to the framed little discs, Tamarkin's proof of formality for $D_{2}$ has been adapted to the operad $f D_{2}$ by Severa [21], and Kontsevich's proof has been adapted to $f D_{2}$ by the present authors in [7]. Both adaptations are relatively straightforward.

Operad formality theorems have significant applications. The formality of $D_{2}$ plays an important role in Tamarkin's proof 25] of Kontsevich's celebrated deformation quantization theorem. The formality of $f D_{2}$ is used in 3 , to construct chain level homotopy BV-algebra structures for topological conformal field theories and for the 2-fold loop space on a space with a circle action, as well as to give a particular solution to the cyclic Deligne conjecture. The formality of $D_{k}$ for $k \geq 4$ is a primary ingredient in the computation of the homology of spaces of long knots in high dimensions 18 .

1.3. Cyclic operad formality. Unlike $D_{k}$, the operad $f D_{k}$ has the additional structure of being homotopy equivalent to a cyclic operad (see [2] for an explicit cyclic model); being a cyclic operad means roughly that the roles of inputs and 
outputs can be interchanged. The present paper was motivated by the question of whether the operad formality of $f D_{2}$ can be made compatible with the cyclic structure. We answer this question in the affirmative. For $k>2$, formality of $f D_{k}$ as an operad or cyclic operad remains an open question. This cyclic formality has an application to computing the cohomology of diffeomorphism groups of 3dimensional handlebodies, as discussed further below.

In section 2 we will recall a convenient cyclic model $f \underline{\mathcal{M}}$ for $f D_{2}$ constructed in terms of certain Borel-Serre type compactified moduli spaces of genus zero curves with marked points that were first introduced by Harvey [12]. The elements of $f \underline{\mathcal{M}}$ are genus zero stable curves decorated with real tangent rays at the marked points and nodes. The operad composition is obtained by gluing stable curves at marked points and tensoring the corresponding rays. This operad was introduced by Kimura-Stasheff-Voronov [14], and it is closely related to the modular operad of Deligne-Mumford compactified moduli spaces of curves whose formality is proved in 9. Moreover $f \underline{\mathcal{M}}$ is homotopy equivalent, as a cyclic operad, to the operad of genus zero Riemann surfaces with boundary, where the operad composition is defined by gluing along boundary components.

The main result of this paper is:

Theorem A. The cyclic operad $C_{*}^{\text {sing }}(f \underline{\mathcal{M}} ; \mathbb{R})$ of singular chains on $f \underline{\mathcal{M}}$ with real coefficients is quasi-isomorphic as a cyclic operad to its homology, $H_{*}(f \underline{\mathcal{M}} ; \mathbb{R})=$ $B V$, which is the Batalin-Vilkovisky operad.

We emphasize that the new and important point of this theorem is that the chain of quasi-isomorphisms is compatible with the cyclic operad structure. In response to this work, Severa has indicated how to show that his formality proof in [21] is in fact also compatible with the cyclic structure.

Rather than check the compatibility of Kontsevich's proof with the cyclic structure, we take a more conceptual approach and build a new manifestly cyclic operad of "projective graph complexes" as a replacement for Kontsevich's graph complexes. The differential is now a combination of both edge deletions and edge contractions. The projective graph complexes do form a cyclic cooperad. Our proof of Theorem A then follows in outline the method used by Kontsevich in the unframed case. The projective graph complexes map onto the cohomology of $f \underline{\mathcal{M}}$, and they also map to the (semi-algebraic) forms on $f \underline{\mathcal{M}}$ by a variation of Kontsevich's map defined by certain configuration space integrals. We prove that both of these maps are quasi-isomorphisms and are compatible with the cyclic cooperad structures. The theorem then follows by dualising.

1.4. Application to 3-dimensional handlebodies. In the forthcoming paper [8], the first author proves that the modular operad generated by the cyclic operad $f \mathcal{M}$ is homotopy equivalent to the modular operad made from $B$ Diffs of handlebodies. This leads to a Bousfield-Kan spectral sequence computing the cohomology of $B \operatorname{Diff}\left(H_{g}\right)$ for $H_{g}$ a handlebody of genus $g$. Theorem $\mathrm{A}$ implies that this spectral sequence degenerates at the $E^{2}$ page. Similarly, Theorem $\mathrm{A}$ implies that the graph homology complex of the Koszul dual of the $B V$ cyclic operad also computes the cohomology of $B \operatorname{Diff}\left(H_{g}\right)$. (Note that formality as an operad rather than a cyclic operad is not enough to imply these results.) 


\section{The FRAmed 2-Discs AS A CYCliC OPERAD}

The framed little 2-discs operad $f D_{2}$ is homotopy equivalent to a cyclic operad (although it is not itself cyclic on the nose). A convenient cyclic model was introduced in [14, §3.4]. We recall that model and discuss some of its properties.

2.1. Cyclic operads and cooperads. Recall that a cyclic operad is an extension of the concept of an operad in which inputs and outputs are on equal footing; this can be formalised by asking that the $\Sigma_{n}$ action on the arity $n$ space extends to an action of $\Sigma_{n+1}$ in a way compatible with the operad composition maps. The notion of a cyclic operad was first introduced by Getzler and Kapranov [6].

In more detail, a cyclic operad in a symmetric monoidal category $\mathcal{C}$ is defined by the following data: a functor $P$ from the category of nonempty finite sets and bijections to $\mathcal{C}$, and for each pair of finite sets $I, J$ with elements $i \in I, j \in J$ a composition morphism,

$$
i^{\circ}{ }_{j}: P(I) \otimes P(J) \rightarrow P(I \sqcup J \backslash\{i, j\}),
$$

natural in $I$ and $J$ and satisfying the following axioms.

- (Associativity) Given finite sets $I, J, K$, and elements $i \in I, j_{1}, j_{2} \in$ $J, k \in K$, the following diagram commutes:

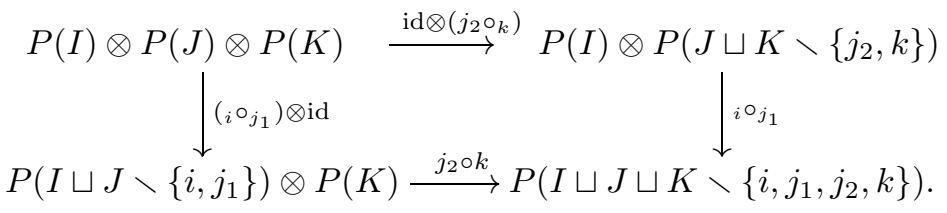

- (Commutativity) Given finite sets $I, J$ and elements $i \in I$ and $j \in J$, the following diagram commutes:

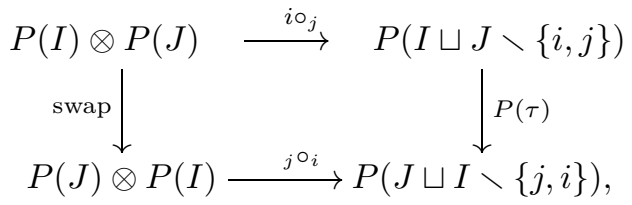

where $\tau$ is the canonical bijection $I \sqcup J \backslash\{i, j\} \cong J \sqcup I \backslash\{j, i\}$.

- (Unit axiom) For each set $A=\{a, b\}$ of cardinality 2 there is a morphism $u_{A}: 1_{\mathcal{C}} \rightarrow P(A)$, where $1_{\mathcal{C}}$ is the monoidal unit, that is natural in $A$ and such that for any finite set $I$ and an element $i \in I$ the following diagram commutes:

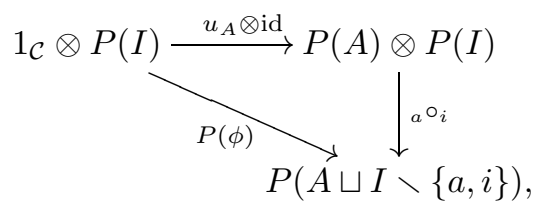

where $\phi: I \cong A \sqcup I \backslash\{a, i\}$ is the canonical bijection sending $i$ to $b$.

Dually one defines a cyclic cooperad by reversing the arrows in the definition. Concretely, a cyclic cooperad is specified by the data of a functor $P$ from finite sets and bijections to $\mathcal{C}$, and a cocomposition map

$$
{ }_{K} \bullet_{L}: P(V) \rightarrow P(K \sqcup\{x\}) \otimes P(L \sqcup\{y\})
$$


(where $x$ and $y$ are auxiliary points) for each partition $V=K \sqcup L$. These cocomposition maps must satisfy dual versions of the naturality, symmetry, and associativity conditions.

A cyclic (co)operad in the category of chain complexes is called a differential graded cyclic (co)operad.

2.2. The compactified genus zero moduli space model. We now recall the moduli space operad introduced by Kimura, Stasheff and Voronov in [14, §3.4]. In their notation, the operad is denoted $\underline{\mathcal{N}}$, but we prefer $f \underline{\mathcal{M}}$ as being more consistent with other notation. This operad is manifestly a cyclic operad, and we will show in Proposition 2.1 that its underlying operad is homotopy equivalent to the framed little 2-discs operad.

Given a nonempty finite set $V$, let $\mathcal{M}_{V}$ denote the moduli space of genus zero Riemann surfaces with marked points labelled by $V$. Explicitly,

$$
\mathcal{M}_{V}:=\operatorname{Inj}\left(V, \mathbb{C P}^{1}\right) / P G L_{2}(\mathbb{C}),
$$

where $\operatorname{Inj}\left(V, \mathbb{C P}^{1}\right)$ is the space of injective maps from $V$ to the complex projective line, and the automorphism group $P G L_{2}(\mathbb{C})$ acts on the target in the standard way. We will write $\mathcal{M}_{n}$ when $V$ is the set $\{0, \ldots, n-1\}$.

There is an identification between $\mathcal{M}_{n}$ and the complement in $\left(\mathbb{C P}^{1}\right)^{n-3}$ of the union of the hyperplanes of the form

$$
z_{i}=z_{j}, z_{i}=0, z_{i}=1, z_{i}=\infty .
$$

If $n>3$, then the moduli space $\mathcal{M}_{n}$ is noncompact and has a Deligne-MumfordKnudsen compactification

$$
\mathcal{M}_{n} \subset \overline{\mathcal{M}}_{n}
$$

which can be constructed by blowing up in a suitable order all proper intersections of hyperplanes of the form (2.2.1) in $\left(\mathbb{C P}^{1}\right)^{n-3}\left[13\right.$. The points of $\overline{\mathcal{M}}_{n}$ correspond to stable nodal Riemann surfaces (nodal means that the surfaces are allowed to have double-point singularities, and stable in this case means that no irreducible component is a sphere with strictly fewer than 3 points that are either nodes or marked points). For $i=0, \ldots, n-1$ there is a complex line bundle $\mathcal{L}_{i}$ over $\overline{\mathcal{M}}_{n}$ whose fibre over a given Riemann surface $S$ is the tangent space at the $i$-th marked point.

The boundary, $\overline{\mathcal{M}}_{V} \backslash \mathcal{M}_{V}$, is a normal crossing divisor. It has an irreducible component for each unordered partition $V=K \sqcup L$ into two subsets of cardinality at least 2 , and the corresponding component is canonically isomorphic to $\overline{\mathcal{M}}_{K \sqcup\{x\}} \times$ $\overline{\mathcal{M}}_{L \sqcup\{y\}}$. The inclusions

$$
\overline{\mathcal{M}}_{K \sqcup\{x\}} \times \overline{\mathcal{M}}_{L \sqcup\{y\}} \rightarrow \overline{\mathcal{M}}_{V}
$$

define a cyclic operad structure on the Deligne-Mumford-Knudsen moduli spaces.

The space $\underline{\mathcal{M}}_{V}$ is defined as the real oriented blowup of the boundary locus of $\overline{\mathcal{M}}_{V}$. We pull the complex line bundles $\mathcal{L}_{x}$ back from $\overline{\mathcal{M}}_{V}$ (and denote them by the same symbol). The space $\underline{\mathcal{M}}_{V}$ can be interpreted as the moduli space of genus zero stable nodal Riemann surfaces with points marked by $V$ and equipped with the additional data of a ray in the tensor product of the two tangent spaces at either side of each node. It can be constructed as the iterated real oriented blowup of $\left(\mathbb{C P}^{1}\right)^{n-3}$ along all intersections of hyperplanes (2.2.1), by blowing up first the proper intersections in the same order as for the Deligne-Mumford space, and 
finally the hyperplanes themselves. The space $\overline{\mathcal{M}}_{V}$ is a compact smooth manifold with faces; i.e., it is a manifold with corners, stratified with the interior as the unique codimension 0 stratum and all of the positive codimension strata lying on the boundary, each of which is a transversal intersection of faces (codimension 1 strata). Faces correspond to unordered partitions of $V$ into two subsets $K \sqcup L$ of cardinality at least 2 ; given such a partition, the corresponding face is canonically isomorphic to the unit circle bundle of the exterior tensor product $\mathcal{L}_{x} \otimes \mathcal{L}_{y}$ over $\underline{\mathcal{M}}_{K \sqcup\{x\}} \times \underline{\mathcal{M}}_{L \sqcup\{y\}}$.

Let $f \mathcal{M}_{V}$ denote the (coarse) moduli space of genus zero smooth surfaces equipped with points marked by $V$ with real tangent rays. Explicitly,

$$
f \mathcal{M}_{V}=\left\{g: V \rightarrow S\left(T \mathbb{C P}^{1}\right) \quad \mid \quad \pi \circ g \in \operatorname{Inj}\left(V, \mathbb{C P}^{1}\right)\right\} / P G L_{2}(\mathbb{C}),
$$

where $\pi: S\left(T \mathbb{C P}^{1}\right) \rightarrow \mathbb{C P}^{1}$ is the circle bundle associated with the tangent bundle of $\mathbb{C P}^{1}$. Observe that there are diffeomorphisms

$$
\begin{aligned}
& f \mathcal{M}_{1} \cong *, \\
& f \mathcal{M}_{2} \cong S^{1} \text { (canonical) }, \text { and } \\
& f \mathcal{M}_{3} \cong\left(S^{1}\right)^{3} \text { (noncanonical) } .
\end{aligned}
$$

In particular, $f \mathcal{M}_{n}$ is compact for $n \leq 3$.

When $n>3, f \mathcal{M}_{n}$ is noncompact. Forgetting the tangent rays at the marked points gives a trivial principal $\left(S^{1}\right)^{n}$-bundle $f \mathcal{M}_{n} \rightarrow \mathcal{M}_{n}$ which extends uniquely to a trivial torus bundle over $\underline{\mathcal{M}}_{n}$. We call its total space $f \underline{\mathcal{M}}_{n} \cong\left(S^{1}\right)^{n} \times \underline{\mathcal{M}}_{n}$. Note that the trivialisation of the bundle is not canonical. More explicitly,

$$
f \underline{\mathcal{M}}_{n}:=S\left(\mathcal{L}_{0}\right) \times \underline{\mathcal{M}}_{n} \cdots \times \underline{\mathcal{M}}_{n} S\left(\mathcal{L}_{n-1}\right) .
$$

One sees that $f \underline{\mathcal{M}}_{n}$ is a compactification of $f \mathcal{M}_{n}$ obtained by adding a boundary and corners, and hence it is homotopy equivalent to its interior. When $n \leq 3$ we simply define $f \underline{\mathcal{M}}_{n}:=f \mathcal{M}_{n}$.

The functor $V \mapsto f \underline{\mathcal{M}}_{V}$ forms a cyclic operad. Composition with $f \underline{\mathcal{M}}_{2} \cong S^{1}$ acts by rotating tangent rays, and composition with $f \underline{\mathcal{M}}_{1} \cong\{*\}$ removes a marked point. All other composition maps are defined by gluing marked points together to form a node and tensoring their tangent rays to produce the decoration at the node; in more detail, if $K$ and $L$ both have cardinality at least 2, then the composition map

$$
{ }_{x}^{\circ}{ }_{y}: f \underline{\mathcal{M}}_{K \sqcup\{x\}} \times f \underline{\mathcal{M}}_{L \sqcup\{y\}} \rightarrow f \underline{\mathcal{M}}_{V}
$$

is a trivial circle bundle over the face associated with the partition $V=K \sqcup L$ and it is induced by the multiplication map $S\left(\mathcal{L}_{x}\right) \times S\left(\mathcal{L}_{y}\right) \rightarrow S\left(\mathcal{L}_{x} \otimes \mathcal{L}_{y}\right)$.

Recall that a homotopy equivalence of topological operads is a morphism of operads (a natural transformation commuting with the composition and unit maps) that is level-wise an equivariant homotopy equivalence.

Proposition 2.1. There is a homotopy equivalence of operads

$$
f D_{2} \simeq f \underline{\mathcal{M}} .
$$

There is also a homotopy equivalence of spaces, $D_{2}(n) \simeq f \underline{\mathcal{M}}_{n+1} /\left(S^{1}\right)^{n}$, where the torus acts by rotating all but the zeroth tangent direction.

Proof. In [19] the second author constructed a zigzag of homotopy equivalences between the operad $D_{2}$ and the Fulton-MacPherson operad, $F M_{2}$, introduced by Getzler and Jones in [4. The space $F M_{2}(n)$ is the real oriented Fulton-MacPherson 
compactification of the space of configurations of $n$ points in $\mathbb{R}^{2}$ modulo dilation and translation. It can be constructed by iterated real oriented blowup from the $(2 n-3)$-sphere $S^{2 n-3} \cong\left(\mathbb{C}^{n} \backslash \Delta(\mathbb{C})\right) / A f f_{2}$, where $\Delta(\mathbb{C})$ is the thin diagonal defined by $z_{1}=\cdots=z_{n}$ and $A f f_{2}:=\mathbb{R}_{+} \ltimes \mathbb{C}$ is the affine group generated by positive dilations and translations. The blowups are performed along all diagonals. The space $F M_{2}(n)$ is a manifold with faces; a face corresponds to an unordered partition of $\{0,1, \ldots, n\}=K \sqcup L$ into two subsets of cardinality at least 2 , and it is canonically diffeomorphic to $F M_{2}(|K|) \times F M_{2}(|L|)$. The inclusion of a face with $L=\{i, \ldots, i+$ $l-1\}$ represents a (noncyclic) $\circ_{i}$ operad composition.

From [20, $D_{2}$ is an operad in the category of spaces with $S O(2)$ action and so one can form the semidirect product operad $D_{2} \ltimes S O(2)$ which is isomorphic to $f D_{2}$. Similarly, $F M_{2}$ is an operad in $S O(2)$-spaces, and one can form the framed Fulton-MacPherson operad $f F M_{2}:=F M_{2} \ltimes S O(2)$. The homotopy equivalence between $D_{2}$ and $F M_{2}$ is realised by a zigzag of $S O(2)$-equivariant maps and thus induces a homotopy equivalence of operads,

$$
f D_{2} \simeq f F M_{2} .
$$

It turns out that $f \mathrm{FM}_{2}$ and $f \underline{\mathcal{M}}$ are isomorphic as operads. This is immediate in arity $n<3$. In higher arity, note that blowing up the sphere $\left(\mathbb{C}^{n} \backslash \Delta(\mathbb{C})\right) / A f f_{2}$ at the diagonal $z_{n-1}=z_{n}$ is the same as blowing up $S^{1} \times\left(\mathbb{C P}^{1}\right)^{n-3}$ at the hypersurfaces $z_{i}=\infty, 1 \leq i \leq n-3$. Blowing up all other diagonals identifies $F M_{2}(n) \cong$ $S^{1} \times \underline{\mathcal{M}}_{n+1}$, and thus we have diffeomorphisms $f F M_{2}(n) \cong f \underline{\mathcal{M}}_{n+1}$ compatible with the operad composition, by definition.

2.3. Semi-algebraic structure and canonical projections. For the proof of Theorem A, we will need to work in the category of semi-algebraic spaces. The theory of semi-algebraic spaces and its corresponding de Rham theory have been developed by Hardt, Lambrechts, Turchin and Volic in [11.

Proposition 2.2. The functor $V \mapsto f \underline{\mathcal{M}}_{V}$ forms a cyclic operad in the category of semi-algebraic manifolds.

Proof. Sinha constructs in 23] a $\Sigma_{n}$-equivariant semi-algebraic embedding $j_{n}$ : $F M_{2}(n) \hookrightarrow\left(S^{1}\right)^{\left(\begin{array}{l}n \\ 2\end{array}\right)} \times[0, \infty]^{\left(\begin{array}{l}n \\ 3\end{array}\right)}$ by evaluating the direction between each pair of points and the ratio of the distances for each triple of points. Thus the symmetric group acts semi-algebraically. Moreover, Lambrechts and Volic check in [17, Prop. 5.5] that the operad composition $\circ_{i}: F M_{2}(n) \times F M_{2}(m) \rightarrow F M_{2}(m+n-1)$ is the restriction of a semi-algebraic map. Passing to the semi-direct product with $S O(2)$, it follows that $f F M_{2}$ is an operad in semi-algebraic manifolds. Finally, we must verify that the action of $\Sigma_{n}$ on $f \underline{\mathcal{M}}_{n} \cong f F M_{2}(n-1)$ is semi-algebraic. For this it is convenient instead to $\Sigma_{n}$-equivariantly embed $f \underline{\mathcal{M}}_{n} \hookrightarrow\left(S^{1}\right)^{\left(\begin{array}{l}n \\ 2\end{array}\right)} \times\left(\mathbb{C P}^{1}\right)^{\left(\begin{array}{l}n \\ 4\end{array}\right)}$ by evaluating angles between rays for pairs of points and the cross ratio for 4-tuples of points. This shows that $\Sigma_{n}$ acts semi-algebraically on $f \underline{\mathcal{M}}_{n}$.

Forgetting subsets of the marked points defines a collection of important projections between the various moduli spaces. Given a subset $A \subset V$, let

$$
\pi^{A}: f \underline{\mathcal{M}}_{V} \rightarrow f \underline{\mathcal{M}}_{V \backslash A}
$$

be the map induced by forgetting all marked points labelled by $A$. Note that $\pi^{A}$ is the iterated operad composition with the point of $f \underline{\mathcal{M}}_{1}$ for each element of $A$. 
Proposition 2.3. The map $\pi^{A}: f \underline{\mathcal{M}}_{V} \rightarrow f \underline{\mathcal{M}}_{V \backslash A}$, forgetting the points in $A$, is a semi-algebraic fibre bundle.

Proof. The projection $\pi^{A}$ is equivalent to the composition $\left(S^{1}\right)^{|V|-1} \times F M_{2}(|V|-$ $1) \rightarrow\left(S^{1}\right)^{|V|-1} \times F M_{2}(|V \backslash A|-1) \rightarrow\left(S^{1}\right)^{|V \backslash A|-1} \times F M_{2}(|V \backslash A|-1)$. The first map is a semi-algebraic bundle by [17, Appendix A], the second map is a trivial bundle, and the composite of bundle projections is a bundle projection by [11, Prop. 8.5].

The fibres of the bundle $\pi^{A}$ are manifolds with faces, so the fibrewise boundary is stratified into pieces of codimension 1 (in the total space) and greater. We shall refer to the codimension 1 strata as the fibrewise boundary components.

Lemma 2.4. For $|V \backslash A| \geq 3$, the fibrewise boundary components of the bundle

$$
\pi^{A}: f \underline{\mathcal{M}}_{V} \rightarrow f \underline{\mathcal{M}}_{V \backslash A}
$$

correspond bijectively with the set of unordered partitions of $V$ into two subsets $U_{1}, U_{2}$ of cardinality at least 2 such that $\left|U_{1} \backslash A\right| \leq 1$.

Proof. Corresponding to a partition $U_{1} \sqcup U_{2}$ is the closure of the locus in $f \underline{\mathcal{M}}_{V}$ in which a node separates the sphere into two lobes, one containing the points of $U_{1}$ and the other containing the points of $U_{2}$. This closed stratum fibres over $f \mathcal{M}_{V \backslash A}$ if and only if one of the $U_{i}$ contains at most one point of $V \backslash A$ (a priori it is an unordered partition, but we can always choose to call the set with this property $\left.U_{1}\right)$.

Given a partition $V=U_{1} \sqcup U_{2}$ as above, we will write $\partial_{U_{1}, U_{2}} \subset f \underline{\mathcal{M}}_{V}$ for the corresponding fibrewise boundary component of $\pi^{A}$.

2.4. Orientations. Fibrewise orientations of the canonical projections play an important role in the formality proof in this paper. Here we establish some general definitions and conventions for working with orientations.

Given a finite-dimensional real vector space $W$, let $\operatorname{det}(W)$ denote the top exterior power of $W$; a choice of a ray in this line is equivalent to a choice of an orientation of $W$. If $S$ is a finite set, then we write $\operatorname{det}(S):=\operatorname{det}\left(\mathbb{R}^{S}\right)($ and $\operatorname{det}(S)=\mathbb{R}$ when $S$ is empty). If $S$ has cardinality at least 2 , then choosing a ray in $\operatorname{det}(S)$ is equivalent to choosing an ordering of $S$ up to even permutations.

An orientation form on an $n$-dimensional manifold $M$ is a nowhere vanishing $n$-form $\Omega_{M}$. Clearly an orientation form determines an orientation of $M$, and two orientation forms determine the same orientation if and only if they agree up to multiplication by a positive scalar function. Given a fibre bundle $\pi: E \rightarrow B$, a fibrewise orientation form is a form on $E$ whose restriction to each fibre is an orientation form.

We will use the following general orientation conventions.

- Given $x \in S$, we define an isomorphism $\iota_{x}$ : $\operatorname{det}(S) \cong \operatorname{det}(S \backslash\{x\})$ by the rule $x \wedge r \mapsto r$ (inverse to the map $(x \wedge-)$ ).

- If $M$ is a manifold with boundary with orientation form $\Omega_{M}$ and $X$ is an outward-pointing normal vector field on $\partial M$, then $\partial M$ is given the induced orientation $\Omega_{\partial M}=\iota_{X} \Omega_{M}$. Thus the Stokes formula is $\int_{M} d \xi=\int_{\partial M} \xi$.

- If $\pi_{1}: E_{1} \rightarrow E_{2}$ and $\pi_{2}: E_{2} \rightarrow B$ are fibre bundles with fibrewise orientation forms $\Omega_{1}$ and $\Omega_{2}$ respectively, then we give the composite bundle $\left(\pi_{2} \circ \pi_{1}\right)$ the fibrewise orientation form $\pi_{1}^{*} \Omega_{2} \wedge \Omega_{1}$. 
Given a finite set $V$ and an element $u \in V$, the canonical projection $\pi^{\{u\}}$ : $f \underline{\mathcal{M}}_{V} \rightarrow f \underline{\mathcal{M}}_{V \backslash\{u\}}$ has a standard fibrewise orientation defined as follows. It factors as

$$
f \underline{\mathcal{M}}_{V} \stackrel{\pi_{\text {dec }}^{\{u\}}}{\longrightarrow} f \underline{\mathcal{M}}_{V} / S^{1} \stackrel{\pi_{\text {pos }}^{\{u\}}}{\longrightarrow} f \underline{\mathcal{M}}_{V \backslash\{u\}},
$$

where $\pi_{\text {dec }}^{\{u\}}$ forgets the tangent ray decoration at the marked point $u$, and $\pi_{\text {pos }}^{\{u\}}$ forgets the position of $u$, thus forgetting it entirely. The principal $S^{1}$-bundle $\pi_{\mathrm{dec}}^{\{u\}}$ inherits a fibrewise orientation $d \theta_{u}$ from the counterclockwise orientation of the circle. The map $\pi_{\text {pos }}^{\{u\}}$ is a family of Riemann surfaces and so the complex structures of the fibres determine a fibrewise orientation $\Omega_{u}$. Using the above orientation conventions, we then give $\pi^{\{u\}}$ the fibrewise orientation determined by the form $\left(\pi_{\mathrm{dec}}^{\{u\}}\right)^{*} \Omega_{u} \wedge d \theta_{u}$.

Given $A \subset V$, we define a bijection between rays in $\operatorname{det}(A)$ and fibrewise orientations of $\pi^{A}: f \underline{\mathcal{M}}_{V} \rightarrow f \underline{\mathcal{M}}_{V \backslash A}$ as follows. The ray spanned by $u_{1} \wedge \cdots \wedge u_{k}$ (where $u_{i}$ are the elements of $A$ ) corresponds to the fibrewise orientation determined by the above conventions and the factorisation $\pi^{A}=\pi^{\left\{u_{1}\right\}} \circ \cdots \circ \pi^{\left\{u_{k}\right\}}$.

\section{Cohomology of the Unframed AND FRAmed LitTle Discs}

We will first recall Arnold's well-known presentation of the cohomology of the ordered configuration spaces of $\mathbb{R}^{2}$, or equivalently of the spaces of the unframed little discs operad $D_{2}$. Then we will use this result to give a new presentation of the cohomology of the framed little discs operad which will be convenient for working with the cyclic operad structure.

3.1. Unframed little 2-discs. Consider the little 2-discs operad $D_{2}$. For $1 \leq i<$ $j \leq k$, consider the map

$$
\pi_{i j}^{\prime}: D_{2}(k) \rightarrow D_{2}(2) \simeq S^{1}
$$

which forgets all but the $i$-th and $j$-th discs. Define a collection of 1 -forms

$$
\omega_{i j}:=\left(\pi_{i j}^{\prime}\right)^{*} d \theta
$$

where $d \theta$ is the standard volume form of $S^{1}$. (We will often abuse notation by using the same symbol for both a differential form and the cohomology class it represents.)

Theorem 3.1 (Arnold). The real cohomology of $D_{2}(n)$ is generated by the degree 1 classes $\omega_{i j}$ for $1 \leq i \neq j \leq n$, subject to only to the symmetry relation,

$$
\omega_{i j}=\omega_{j i}
$$

and the Arnold relation,

$$
\omega_{i j} \omega_{j k}+\omega_{j k} \omega_{k i}+\omega_{k i} \omega_{i j}=0
$$

for each triple of distinct indices $\{i, j, k\}$.

In fact, this gives a presentation of the integral cohomology, but we shall only be concerned with real cohomology in this paper. 
3.2. Framed little 2-discs. Since $f \underline{\mathcal{M}}_{n+1} \simeq f D_{2}(n)=D_{2}(n) \times\left(S^{1}\right)^{n}$, where the $i$-th $S^{1}$ factor measures the angle by which the $i$-th disc is rotated, one has

$$
H^{*}\left(f \underline{\mathcal{M}}_{n+1}\right) \cong H^{*}\left(D_{2}(n)\right) \otimes \Lambda\left(\eta_{1}, \ldots, \eta_{n}\right)
$$

where $\eta_{i}$ is the class represented by the volume form $d \theta_{i}$ of the $i$-th circle. Thus the cohomology is generated by the classes $\omega_{i j}$ together with the classes $\eta_{i}$, and subject only to the symmetry and Arnold relations. The symmetric group $\Sigma_{n+1}$ acts on $f \underline{\mathcal{M}}_{n+1}$ by permuting the labels of the marked points. The subgroup $\Sigma_{n} \subset \Sigma_{n+1}$, consisting of permutations which fix 0 , permutes the generators and relations among themselves in the obvious way. However, the permutations which do not fix zero act in a more complicated way.

We will now give a different presentation in which the generators and relations of $H^{*}\left(f \underline{\mathcal{M}}_{n+1}\right)$ are both permuted by the full symmetric group $\Sigma_{n+1}$. Let $\pi_{i j}: f \underline{\mathcal{M}}_{n+1} \rightarrow f \underline{\mathcal{M}}_{2}$ be the projection forgetting all but the $i$-th and the $j$-th points with their tangent rays (see earlier section 2.3). Since $f \underline{\mathcal{M}}_{2} \cong S^{1}$, we define closed 1-forms

$$
\alpha_{i j}:=\pi_{i j}^{*} d \theta \in \Omega^{*}\left(f \underline{\mathcal{M}}_{n+1}\right)
$$

which represent generators of the cohomology. To describe the relations among these generators we must relate them to the generators $\omega_{i j}, \eta_{i}$ described above.

There are two ways of identifying the space $f \underline{\mathcal{M}}_{3}$ with $\left(S^{1}\right)^{3}$. The first way is to apply the unique conformal automorphism of $\mathbb{C} P^{1}$ which puts the marked points $p_{0}, p_{1}, p_{2}$ labelled by $0,1,2$ at the points $\infty, 0,1 \in \mathbb{C} P^{1}$ respectively. One then reads off the angle $\varphi_{i}$, in the counterclockwise sense, from the equator (going in the direction from 0 to 1 to $\infty$ ) to the tangent ray at the $i$-th marked point. In terms of these coordinates, $\alpha_{i j}=d\left(\varphi_{i}+\varphi_{j}\right)$.

The other way of identifying $f \underline{\mathcal{M}}_{3}$ with $\left(S^{1}\right)^{3}$ is to put the points at $\infty, 0,1$ and then rotate the sphere around the axis through 0 and $\infty$, so that the tangent ray of the point at $\infty$ is parallel to the equator (pointing in the direction from $\infty$ to 0 to 1 ). We define $\theta_{1}$ to be the angle between the ray at 0 and the equator. Parallel transport along the geodesic from $p_{1}$ to $p_{2}$ of the tangent ray to the equator at $p_{1}$ gives a reference ray at $p_{2}$ and $\theta_{2}$ is the angle from this ray to the tangent ray of $p_{2}$. The angle $\psi$ is measured at $p_{1}$ from the equator to the geodesic from $p_{1}$ and $p_{2}$. In terms of these coordinates, $\omega_{12}=d \psi$ and $\eta_{i}=d \theta_{i}$.

These two different coordinate systems are illustrated below.
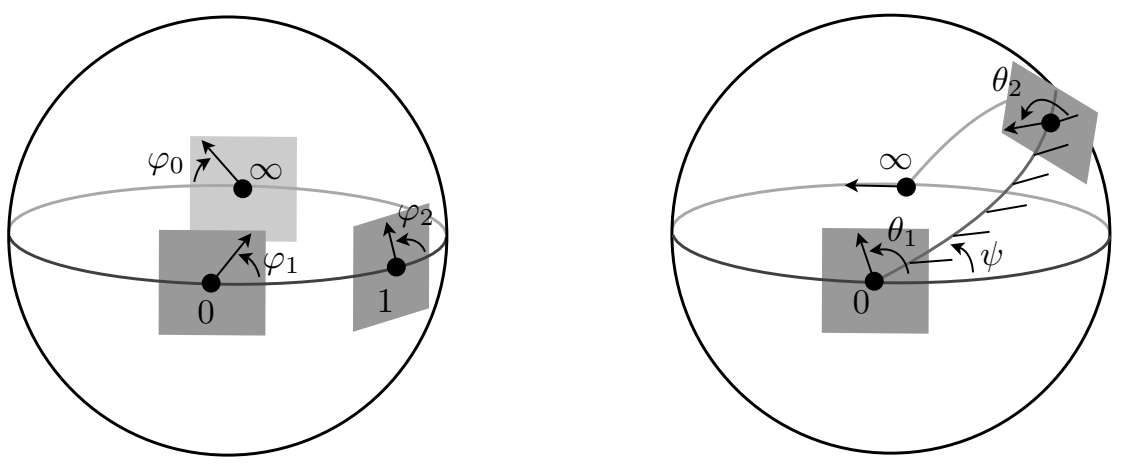
One easily sees that these two coordinate systems are related as follows:

$$
\begin{array}{rlrl}
\theta_{1} & =\varphi_{1}+\varphi_{0}, & \varphi_{1}=\theta_{1}-\psi, \\
\theta_{2} & =\varphi_{2}+\varphi_{0}, & & \varphi_{2}=\theta_{2}-\psi, \\
\psi & =\varphi_{0} . &
\end{array}
$$

Hence, in the cohomology of $f \underline{\mathcal{M}}_{n}$ the two sets of generators are related by:

$$
\begin{array}{rlrl}
\omega_{i j} & =(1 / 2)\left(\alpha_{0 i}+\alpha_{0 j}-\alpha_{i j}\right), & \alpha_{i j}=\eta_{i}+\eta_{j}-2 \omega_{i j}, \\
\eta_{i} & =\alpha_{0 i} .
\end{array}
$$

In terms of the $\alpha$ classes, the Arnold relation becomes:

$$
\begin{aligned}
& \quad \alpha_{i j} \alpha_{j k}+\alpha_{j k} \alpha_{k i}+\alpha_{k i} \alpha_{i j} \\
& +\alpha_{i 0} \alpha_{0 j}+\alpha_{j 0} \alpha_{0 k}+\alpha_{k 0} \alpha_{0 i} \\
& +\alpha_{k j} \alpha_{j 0}+\alpha_{i k} \alpha_{k 0}+\alpha_{j i} \alpha_{i 0} \\
& +\alpha_{0 j} \alpha_{j i}+\alpha_{0 k} \alpha_{k j}+\alpha_{0 i} \alpha_{i k}=0 .
\end{aligned}
$$

The action of the symmetric group must send relations to relations, so one can write the relations in a more invariant form. Let $\left\{i_{1}, i_{2}, i_{3}, i_{4}\right\}$ be four distinct elements of $\{0, \ldots, n\}$. The cyclic Arnold relations are

$$
\sum_{\sigma \in A_{4}} \alpha_{i_{\sigma(1)} i_{\sigma(2)}} \alpha_{i_{\sigma(2)} i_{\sigma(3)}}=0
$$

where $A_{4}$ is the alternating group on 4 letters. Clearly the symmetric group $\Sigma_{n+1}$ sends each relation of this type to another relation of this type.

Theorem 3.2. The real cohomology of $f \underline{\mathcal{M}}_{n+1}$ is generated by the 1-dimensional classes $\alpha_{i j}(0 \leq i \neq j \leq n)$ subject only to the cyclic Arnold relations, one for each 4-tuple of distinct indices $\left\{i_{1}, i_{2}, i_{3}, i_{4}\right\}$ between 0 and $n$, and to the symmetry relation $\alpha_{i j}=\alpha_{j i}$.

\section{The AFFine AND PROJECTIVE GRAPH COMPleXeS}

The main ingredient in Kontsevich's proof of formality of the unframed little discs operad is the construction of a certain differential graded algebra of graphs, the Kontsevich graph complex $\mathcal{K G}_{n}$, which resolves the usual Arnold presentation (Theorem 3.1) of the cohomology of the little discs $D_{2}(n)$. As $n$ varies, these DGAs of graphs fit together to form a cooperad, and the dual operad is quasi-isomorphic to the operad of chains on the little discs.

Tensoring Kontsevich's resolution $\mathcal{K G}_{n}$ of $H^{*}\left(D_{2}(n)\right)$ with $\Lambda\left(\eta_{1}, \ldots, \eta_{n}\right)$ gives a resolution of the presentation (3.2.1) of the cohomology ring of $f D_{2}(n) \simeq f \underline{\mathcal{M}}_{n+1}$; we shall refer to this DGA as the affine graph complex, denoted $\mathcal{A G}_{n}$. The collection of DGAs $\left\{\mathcal{A G}_{n}\right\}$ forms a cooperad, but it is not compatible with the cyclic operad structure of $f \underline{\mathcal{M}}$. To deal with this we will pass to the projective graph complex $\mathcal{P} \mathcal{G}_{n+1}$. The projective graph complexes do form a cyclic cooperad, and we prove that $\mathcal{P} \mathcal{G}_{n+1}$ is indeed a resolution of $H^{*}\left(f \underline{\mathcal{M}}_{n+1}\right)$ by a comparison with $\mathcal{A} \mathcal{G}_{n}$. Summarising this:

- $\mathcal{K} \mathcal{G}_{n}$ is Kontsevich's graph complex; it resolves $H^{*}\left(D_{2}(n)\right)$ over $\mathbb{R}\left[\Sigma_{n}\right]$.

- $\mathcal{A G}_{n}=\mathcal{K G}_{n} \otimes \Lambda\left(\eta_{1}, \ldots, \eta_{n}\right)$ is the affine graph complex; it resolves $H^{*}\left(f \underline{\mathcal{M}}_{n+1}\right)$ over $\mathbb{R}\left[\Sigma_{n}\right]$. 
- $\mathcal{P} \mathcal{G}_{n+1}$ is the projective graph complex, which resolves $H^{*}\left(f \underline{\mathcal{M}}_{n+1}\right)$ over $\mathbb{R}\left[\Sigma_{n+1}\right]$.

Each of these complexes will be defined in detail below.

4.1. Some preliminaries on graphs and orientations of graphs. By a graph $\gamma=\left(V, V_{\text {int }}, E\right)$ we shall mean a finite set $V \sqcup V_{\text {int }}$ of vertices and a set $E$ of unordered pairs of vertices representing the edges. Note that our graphs cannot have loops or double edges. Those vertices in $V_{\text {int }}$ are called internal vertices, and those in $V$ are called external vertices; there is an induced partition of the set of edges

$$
E=E_{\text {ext }} \sqcup E_{\partial} \sqcup E_{\text {int }},
$$

where the set of internal edges, $E_{\text {int }}$, consists of those edges for which both endpoints are internal, the set of external edges, $E_{\text {ext }}$, consists of those for which both endpoints are external vertices, and the set of boundary edges, $E_{\partial}$, consists of those which have one internal endpoint and one external endpoint.

Definition 4.1. An affine orientation of a graph $\gamma=\left(V, V_{i n t}, E\right)$ is a choice of a ray in the real line $\operatorname{det}(E)$. A projective orientation of $\gamma$ is a choice of a ray in $\operatorname{det}\left(V_{\text {int }} \sqcup E\right)$.

If $\gamma$ is equipped with an affine or projective orientation, then we shall write $\bar{\gamma}$ for the same underlying graph equipped with the opposite orientation.

4.1.1. Edge deletion. Given a graph $\gamma=\left(V, V_{\text {int }}, E\right)$ and an edge $e$, we define a new graph $\gamma \backslash e=\left(V, V_{\text {int }}, E \backslash\{e\}\right)$ by deleting the edge $e$. If $\gamma$ has a projective or affine orientation, then $\gamma \backslash e$ inherits an orientation of the same type by the isomorphism $\iota_{e}$ from section 2.4

4.1.2. Edge contraction. Given a graph $\gamma=\left(V, V_{i n t}, E\right)$ and an edge $e$ that is not part of a triangle of edges and has at least one internal endpoint, let $\gamma / e$ denote the graph constructed from $\gamma$ by contracting the edge $e$, i.e. deleting $e$ from the set of edges and identifying its two endpoints together. If $e$ is a boundary edge, then the resulting vertex is external; if $e \in E_{\text {int }}$, then the resulting vertex is internal. Thus the set of external vertices of $\gamma / e$ is identified with $V$.

If $\gamma$ has an affine orientation, then $\gamma / e$ inherits an affine orientation by the isomorphism $\iota_{e}$. Inducing projective orientations is slightly more complicated and depends on whether $e$ is boundary or internal.

- If $e \in E_{\partial}$, then one endpoint, $u$, is external and one endpoint, $v$, is internal. In this case there is a canonical identification of the edges of $\gamma / e$ with $E \backslash$ $\{e\}$; we identify the external vertices of $\gamma / e$ with $V$, and the internal vertices with $V_{\text {int }} \backslash\{v\}$, by identifying the vertex in $\gamma / e$ at which $e$ was contracted with $u$. Using these identifications, the induced projective orientation of $\gamma / e$ is the image of the projective orientation of $\gamma$ under the isomorphism $\iota_{v} \circ \iota_{e}$.

- If $e \in E_{i n t}$, then inducing a projective orientation on $\gamma / e$ is equivalent to choosing an orientation of $e$, meaning a choice of one end, $v$, as head and the other, $u$, as tail. As in the boundary edge case, we contract $e$ onto its tail and identify the internal vertices of $\gamma / e$ with $V \backslash\{v\}$, and then we induce a projective orientation via $\iota_{v} \circ \iota_{e}$. 
4.1.3. Combined deletion and contraction. Suppose that $(e, f)$ is a pair of edges in a graph $\gamma$ meeting at an internal vertex $u$ and such that $e$ is not a side of a triangle in $\gamma \backslash f$. Then we shall write

$$
\gamma \oslash(e, f):=(\gamma \backslash f) / e .
$$

If $\gamma$ has a projective orientation and $e$ is either a boundary edge or an oriented internal edge, then $\gamma \oslash(e, f)$ inherits a projective orientation by the above discussion.

4.2. The affine and projective graph complexes. First recall Kontsevich's complex of "admissible graphs" [15, Definition 13], denoted $\mathcal{K} \mathcal{G}$.

Definition 4.2. The vector space $\mathcal{K G}_{V}$ is spanned by isomorphism classes of affine oriented graphs with external vertex set $V$ modulo the relations

(1) $\gamma \sim 0$ if there is a connected component of $\gamma$ containing no external vertices,

(2) $\gamma \sim 0$ if there is an internal vertex of valence $<3$,

(3) $\bar{\gamma} \sim-\gamma$.

There is a grading defined by $|E|-2\left|V_{\text {int }}\right|$. The differential of degree 1 is given by the formula

$$
d \gamma=\sum_{e} \gamma / e
$$

where $e$ runs among those boundary and internal edges such that $\gamma / e$ is defined. There is an associative and graded commutative product defined by gluing the external vertices together according to the labelling, and by tensoring orientation rays.

Note that the differential is a derivation of the product, and so $\mathcal{K} \mathcal{G}_{V}$ forms a commutative DGA. We will write $\mathcal{K G}_{V}=\mathcal{K G}_{n}$ when $V=\{1, \ldots, n\}$.

Definition 4.3. The affine graph complex is the CDGA defined as

$$
\mathcal{A G}_{n}:=\mathcal{K} \mathcal{G}_{n} \otimes \Lambda\left(\eta_{1}, \ldots, \eta_{n}\right) .
$$

We now come to the principal object of this paper: the CDGA of projective graphs.

Definition 4.4. The vector space of projective graphs $\mathcal{P} \mathcal{G}_{V}$, for a finite set $V$, is spanned by isomorphism classes of projectively oriented graphs $\gamma$ with $V$ as the set of external vertices, modulo the following relations:

$(R 1)$ if $\gamma$ has an internal vertex of valence $\leq 3$, then $\gamma \sim 0$,

$(R 2)$ if $\gamma$ has a component with at most one external vertex and at least one internal vertex, then $\gamma \sim 0$,

(R3) $\bar{\gamma} \sim-\gamma$,

$(R 4)$ the "pinwheel relation": for $v$ an internal vertex of $\gamma$ and $E(v)$ the set of edges incident at $v$,

$$
\sum_{e \in E(v)}(\gamma \backslash e) \sim 0
$$

There is a grading defined by $\operatorname{deg}(\gamma):=|E|-3\left|V_{\text {int }}\right|$. There is an associative and graded commutative algebra structure on $\mathcal{P} \mathcal{G}_{V}$ given by gluing at the external vertices, and tensoring orientation rays. 
In the case $V=\{0, \ldots, n-1\}$, we write $\mathcal{P} \mathcal{G}_{V}=\mathcal{P} \mathcal{G}_{n}$.

We next define a differential $d$ on $\mathcal{P G}_{V}$. The formula will involve choices, but we will show that because of the pinwheel relation it is in fact independent of these choices. To define $d \gamma$, with $\gamma \in \mathcal{P} \mathcal{G}_{V}$, we first arbitrarily choose an orientation of each internal edge $e$ of $\gamma$. We orient the boundary edges in each case so that their heads are at their internal endpoints. The differential is now given by the formula

$$
d \gamma=\sum_{(e, f)} \gamma \oslash(e, f)
$$

where the sum runs over all ordered pairs of edges $(e, f)$ for which the head of $e$ is internal and incident to $f$ (so, in particular, they must lie in $E_{\partial} \sqcup E_{\text {int }}$ ) and such that $\gamma \oslash(e, f)$ is defined (i.e. deleting $f$ and contracting $e$ does not result in a loop or double-edge).

Proposition 4.5. $d \gamma$ is well-defined, independent of the choices of orientations for the edges between internal vertices.

Proof. Suppose $e$ is an edge between internal vertices $v, w$.

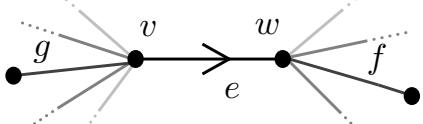

The difference between $d \gamma$ with $e$ oriented one way or the other is precisely the pinwheel relation in $\gamma / e$ at the vertex where $e$ is contracted. Thus $d \gamma$ is independent of the choices of orienting the internal edges.

It remains to check that $d$ descends to the quotient by the pinwheel relation. Given a graph $\gamma$ with an internal vertex $v$, orient all of the (internal) edges of $E(v)$ with their heads at $v$. Then,

$$
\begin{aligned}
d\left(\sum_{g \in E(v)} \gamma \backslash g\right)=\sum_{g \in E(v)} \sum_{\substack{(e, f) \operatorname{in} \gamma \backslash g\\
}}(\gamma \backslash g) \oslash(e, f) \\
=\sum_{g \in E(v)} \sum_{\substack{(e, f) \text { in } \gamma \backslash g \\
e \notin E(v)}}-(\gamma \oslash(e, f) \backslash g)+\sum_{g \in E(v)} \sum_{\substack{(e, f) \text { in } \gamma \backslash g \\
e \in E(v)}}-(\gamma \oslash(e, f) \backslash g) .
\end{aligned}
$$

The first of the above two summations can be grouped as a sum of terms, each of which is a pinwheel sum over edges $g$ incident at $v$ in $\gamma \oslash(e, f)$. The terms of the second summation cancel in pairs since $e \in E(v)$ oriented with head at $v$ means that $f \in E(v)$, and $\gamma \oslash(e, f) \backslash g=-\gamma \oslash(e, g) \backslash f$.

Proposition 4.6. $d^{2}=0$.

Proof. To compute $d^{2} \gamma$ we first choose an orientation of each internal edge of $\gamma ; d \gamma$ is a sum of terms of the form $\gamma \oslash(e, f)$ and the internal edges of each of these terms inherit orientations. Now, $d^{2} \gamma$ is a sum of graphs of the form $\pm \gamma \oslash(e, f) \oslash\left(e^{\prime}, f^{\prime}\right)$ (the sign depends only on $\gamma$ ), where $e, f$ are internal or boundary edges in $\gamma$ with $f$ incident at the head of $e$, and $e^{\prime}, f^{\prime}$ are internal or boundary edges in $\gamma \oslash(e, f)$ 
with $f^{\prime}$ incident at the head of $e^{\prime}$. This means that, as edges in $\gamma$, the possible configurations are enumerated as follows:

Type (0): The head of $e^{\prime}$ is incident at $f^{\prime}$ in $\gamma$ and the heads of $e$ and $e^{\prime}$ are disjoint.

If the head of $e^{\prime}$ is incident at $f^{\prime}$ in $\gamma$ but the configuration is not of type (0) then it must be

Type (1): $f, f^{\prime}, e$ and $e^{\prime}$ all meet at a vertex.

If the head of $e^{\prime}$ is not incident at $f^{\prime}$ in $\gamma$, then it must be incident at $f^{\prime}$ in $\gamma \oslash(e, f)$. This can happen only if, in $\gamma$, either $e^{\prime}$ and $f^{\prime}$ are incident at opposite ends of $e$ (types (2), (4), and (5) below), or if $f^{\prime}$ is incident at the tail of $e^{\prime}$ and contracting $e$ causes $e^{\prime}$ to reverse orientation (type (3) below). So the configuration must be one of the following types.

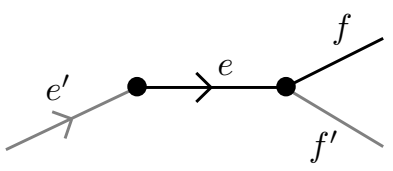

Type (2)

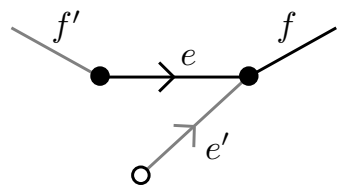

Type (4)

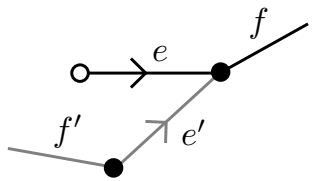

Type (3)

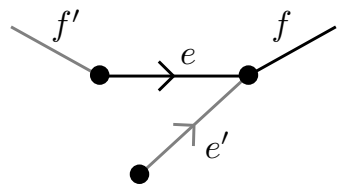

Type (5)

o : external vertex

- : internal vertex

unmarked: either internal or external vertex

We will show case by case that all terms can be grouped to exactly cancel. For type (0), the way that orientations are induced yields

$$
\gamma \oslash(e, f) \oslash\left(e^{\prime}, f^{\prime}\right)=\overline{\gamma \oslash\left(e^{\prime}, f^{\prime}\right) \oslash(e, f)},
$$

and so these two terms in $d^{2} \gamma$ cancel. For types (1) and (2), $\gamma \oslash(e, f) \oslash\left(e^{\prime}, f^{\prime}\right)$ cancels with $\gamma \oslash\left(e, f^{\prime}\right) \oslash\left(e^{\prime}, f\right)$. In the configuration of type $(3), \gamma \oslash(e, f) \oslash\left(e^{\prime}, f^{\prime}\right)$ cancels with $\gamma \oslash\left(e^{\prime}, f\right) \oslash\left(e, f^{\prime}\right)$, which is a term of type (4), and hence the terms of type (3) cancel in pairs with the terms of type (4).

Type (5) is slightly more involved. By the pinwheel relation,

$$
\begin{aligned}
\sum_{f^{\prime} \in E(u) \backslash e} \gamma \oslash(e, f) \oslash\left(e^{\prime} f^{\prime}\right)= & -\sum_{g \in E(v) \backslash\left\{e, f, e^{\prime}\right\}} \gamma \oslash(e, f) \oslash\left(e^{\prime}, g\right) \\
& +\sum_{h \in E(w) \backslash e^{\prime}} \gamma \oslash(e, f) \oslash\left(\overline{e^{\prime}}, h\right) .
\end{aligned}
$$



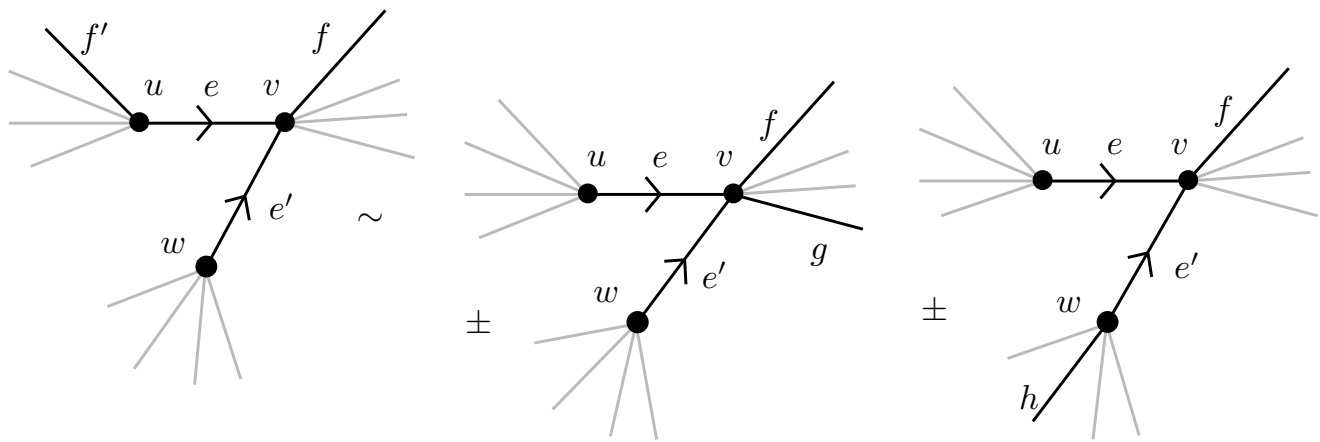

Consider the sum of the expression (4.2.1) over all $f \in E(v) \backslash\left\{e, e^{\prime}\right\}$; the sum over $f, g$ is zero because of the symmetry of swapping $f$ and $g$ (just as for type (0)), and the sum over $f, h$ exactly cancels with the sum over $f, h$ of the type (5) terms $\gamma \oslash\left(e^{\prime}, f\right) \oslash(e, h)$ occurring in $d^{2} \gamma$.

4.3. The combinatorial 'integration' maps. Here we introduce maps of graph complexes that are combinatorial analogues of certain configuration space integrals.

Fix finite sets $V, V_{\text {int }}$. Let $\mathcal{P} \mathcal{G}_{V \sqcup V_{i n t}}^{0} \subset \mathcal{P} \mathcal{G}_{V \sqcup V_{i n t}}$ denote the subalgebra of graphs having no internal vertices. Note that for such a graph $\gamma=\left(V \sqcup V_{\text {int }}, \emptyset, E\right)$, a projective orientation is simply a ray in $\operatorname{det}(E)$. Given a ray $r \subset \operatorname{det}\left(V_{\text {int }}\right)$, there is a linear map

$$
\pi_{r}^{V_{i n t}}: \mathcal{P G}_{V \sqcup V_{i n t}}^{0} \rightarrow \mathcal{P} \mathcal{G}_{V}
$$

It is defined by turning the $V_{\text {int }}$ vertices into internal vertices and mapping the orientations by

$$
\operatorname{det}(E) \stackrel{-\wedge r}{\longrightarrow} \operatorname{det}\left(V_{\text {int }} \sqcup E\right) .
$$

Note that $\pi_{-r}^{V_{i n t}}=-\pi_{r}^{V_{i n t}}$. Geometrically, this map corresponds to integrating out the $V_{\text {int }}$ vertices as we shall see in section 6 .

A general projectively oriented graph $\gamma \in \mathcal{P} \mathcal{G}_{V}$ with a set of internal vertices $V_{\text {int }}$ can be written as $\gamma=\pi_{r}^{V_{\text {int }}}(\widetilde{\gamma})$, where $\widetilde{\gamma} \in \mathcal{P} \mathcal{G}_{V \sqcup V_{\text {int }}}^{0}$ and $r$ is a ray in $\operatorname{det}\left(V_{\text {int }}\right)$.

\subsection{The cyclic cooperad structure on the projective graph complexes.}

We now define a cyclic cooperad structure on the collection of projective graph complexes. For any partition $V=I \sqcup J$ we need to define a cocomposition map

$$
{ }_{I} \bullet_{J}: \mathcal{P} \mathcal{G}_{V} \rightarrow \mathcal{P} \mathcal{G}_{I \sqcup\left\{x_{1}\right\}} \otimes \mathcal{P} \mathcal{G}_{J \sqcup\left\{x_{2}\right\}} .
$$

Let $e_{a, b}$ be the graph consisting of a single edge between external vertices $a$ and $b$, oriented from $a$ to $b$. Then,

$$
\left({ }_{I} \bullet_{J}\right)\left(e_{a, b}\right)= \begin{cases}e_{a, b} \otimes 1 & \text { if } a, b \in I, \\ 1 \otimes e_{a, b} & \text { if } a, b \in J, \\ e_{a, x_{1}} \otimes 1+1 \otimes e_{x_{2}, b} & \text { if } a \in I \text { and } b \in J .\end{cases}
$$

A graph $\gamma \in \mathcal{P} \mathcal{G}_{V}^{0}$ can be written as a product of graphs each having only a single edge. The order of the factors corresponds to the chosen orientation. The multiplication of the formulae above for the edges of $\gamma$ defines $\left({ }_{I} \bullet J\right)(\gamma)$. 
Given a partition $V_{\text {int }}=A \sqcup B$, choose rays $s \in \operatorname{det}(A)$ and $t \in \operatorname{det}(B)$ such that $s \wedge t=r$. Now consider the composition

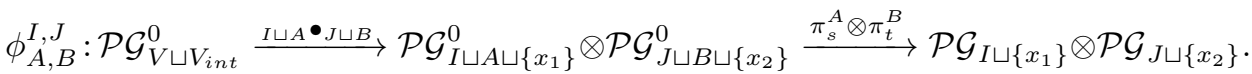

We can now define the cooperad cocomposition map on an arbitrary graph $\gamma=$ $\pi_{r}^{V_{\text {int }}}(\widetilde{\gamma})$ by the formula

$$
\left({ }_{I} \bullet J\right)(\gamma):=\sum_{V_{\text {int }}=A \sqcup B} \phi_{A, B}^{I, J}(\widetilde{\gamma})
$$

There is a useful alternative description of this cocomposition that will make the verification of certain properties easier. An $(I, J)$-splitting of a graph $\gamma$ is a partition of the vertices into two sets $V_{L} \sqcup V_{R}=V \sqcup V_{\text {int }}$ with $I \subset V_{L}$ and $J \subset V_{R}$ and a partition of the edges into two sets $E_{L} \sqcup E_{R}$ such that if both of the endpoints of an edge $e$ are in $V_{i}(i \in\{L, R\})$, then $e \in E_{i}$. Given a splitting $\tau$, we can construct two new graphs $\gamma_{i}^{\tau}$ with vertices $V_{i} \sqcup\left\{x_{i}\right\}$ and edges $E_{i}$; if $e \in E_{i}$, as an edge in $\gamma$, has only one endpoint in $V_{i}$, then as an edge in $\gamma_{i}^{\tau}$ its endpoint that is not in $V_{i}$ is replaced by the auxiliary vertex $x_{i}$. If $\gamma$ has a projective orientation $\Omega$, then we give $\gamma_{L}^{\tau}$ and $\gamma_{R}$ projective orientations $\Omega_{L}$ and $\Omega_{R}$ such that $\Omega_{L} \wedge \Omega_{R}=\Omega$. By inspection one finds the following.

Proposition 4.7. The cocomposition map defined above in 4.4.1) is given by the formula

$$
{ }_{I} \bullet_{J}(\gamma)=\sum_{\tau \in S(\gamma)} \gamma_{L}^{\tau} \otimes \gamma_{R}^{\tau}
$$

where the sum runs over the set $S(\gamma)$ of all $(I, J)$-splittings of $\gamma$.

Proposition 4.8. The cocomposition maps given above are well-defined and they make $\mathcal{P} \mathcal{G}_{V}$ into a cyclic cooperad in the category of commutative differential graded algebras.

Proof. There are three facts to verify.

(i) ${ }_{I} \bullet J$ is compatible with the relations $(R 1-4)$ of Definition 4.4

(ii) ${ }_{I} \bullet_{J}$ commutes with the differential.

(iii) These maps satisfy the cyclic cooperad axioms given in section 2.1.

For (i), it is easy to verify compatibility with $(R 1-3)$. For the pinwheel relation $(R 4)$, suppose $\gamma$ is a projective graph with an internal vertex $v$ and external vertices $I \sqcup J$. Then

$$
\begin{aligned}
I_{J}\left(\sum_{f \in E(v)} \gamma \backslash f\right) & =\sum_{f \in E(v)}{ }_{I}{ }_{J}(\gamma \backslash f) \\
& =\sum_{f \in E(v)} \sum_{\tau} \gamma_{L}^{\tau} \otimes \gamma_{R}^{\tau}
\end{aligned}
$$


where $\tau$ runs over the set $S(\gamma \backslash f)$ of $(I, J)$-splittings of $\gamma \backslash f$. Then (4.4.2) can be written as

$$
\begin{aligned}
& \sum_{f \in E(v)}\left(\sum_{\substack{\tau \in S(\gamma \backslash f) \\
\text { s.t. } v \in V_{L}}} \gamma_{L}^{\tau} \otimes \gamma_{R}^{\tau}+\sum_{\substack{\tau \in S(\gamma \backslash f) \\
\text { s.t. } v \in V_{R}}} \gamma_{L}^{\tau} \otimes \gamma_{R}^{\tau}\right) \\
= & \sum_{f \in E(v)}\left(\sum_{\substack{\tau^{\prime} \in S(\gamma) \\
\text { s.t. } v \in V_{L} \\
\text { and } f \in E_{L}}}\left(\gamma_{L}^{\tau^{\prime}} \backslash f\right) \otimes \gamma_{R}^{\tau^{\prime}}+(-1)^{\operatorname{deg}\left(\gamma_{L}^{\tau^{\prime}}\right)} \sum_{\substack{\tau^{\prime} \in S(\gamma) \\
\text { s.t. } v \in V_{R} \\
\text { and } f \in E_{R}}} \gamma_{L}^{\tau^{\prime}} \otimes\left(\gamma_{R}^{\left.\tau^{\prime} \backslash f\right)}\right)\right. \\
= & \sum_{\substack{\tau^{\prime} \in S(\gamma) \\
\text { s.t. } v \in V_{L}}} \sum_{\substack{f \in E_{L} \cap E(v) \\
\left(\gamma_{L}^{\tau^{\prime}} \backslash f\right) \otimes \gamma_{R}^{\tau^{\prime}}}}+\quad(-1)^{\operatorname{deg}\left(\gamma_{L}^{\tau^{\prime}}\right)} \sum_{\substack{\tau^{\prime} \in S(\gamma) \\
\text { s.t. } v \in V_{R}}} \sum_{f \in E_{R} \cap E(v)} \gamma_{L}^{\tau^{\prime}} \otimes\left(\gamma_{R}^{\tau^{\prime}} \backslash f\right) .
\end{aligned}
$$

This verifies statement (i).

We turn to (ii) now. Given an oriented edge $e$, let $h(e)$ denote the head and $t(e)$ the tail. Observe that choosing a splitting of $\gamma \oslash(e, f)$ is the same as choosing a splitting of $\gamma$ such that $f, h(e), t(e)$ are either all on the left or all on the right. Hence,

$$
\begin{aligned}
& { }_{I}{ }_{J}(d \gamma)=\sum_{(e, f) \text { in } \gamma}\left(\sum_{\tau \in S(\gamma \oslash(e, f))} \gamma_{L}^{\tau} \otimes \gamma_{R}^{\tau}\right) \\
& =\sum_{(e, f) \text { in } \gamma}\left(\sum_{\begin{array}{c}
\tau^{\prime} \in S(\gamma) \\
\text { s.t. } e, f \in E_{L} \\
h(e), t(e) \in V_{L}
\end{array}}\left(\gamma_{L}^{\tau^{\prime}} \oslash(e, f)\right) \otimes \gamma_{R}^{\tau^{\prime}}+(-1)^{\operatorname{deg}\left(\gamma_{L}^{\tau^{\prime}}\right)} \sum_{\begin{array}{r}
\tau^{\prime} \in S(\gamma) \\
\text { s.t. } e, f \in E_{R} \\
h(e), t(e) \in V_{R}
\end{array}} \gamma_{L}^{\tau^{\prime}} \otimes\left(\gamma_{R}^{\tau^{\prime}} \oslash(e, f)\right)\right) \text {. }
\end{aligned}
$$

Applying the differential to a cocomposition and expanding similarly, one sees that $d\left({ }_{I} \bullet J(\gamma)\right)$ is given by the above sum but without the requirement that $t(e)$ be on the same side as $h(e)$. We will show that these additional terms cancel out. Suppose we are given a pair $(e, f)$ in $\gamma$ meeting at an internal vertex $v=h(e)$ and a splitting $\tau \in S(\gamma)$ such that $t(e) \in V_{L}, h(e) \in V_{R}$, and $e, f \in E_{R}$. Define a new splitting $\tau^{\prime} \in S(\gamma)$ by switching $e, f$ and $h(e)$ from the right to the left. Then

$$
\left(\gamma_{L}^{\tau^{\prime}} \oslash(f, e)\right) \otimes \gamma_{R}^{\tau^{\prime}}=(-1)^{\operatorname{deg}\left(\gamma_{L}^{\tau}\right)+1}\left(\gamma_{L}^{\tau} \otimes\left(\gamma_{R}^{\tau} \oslash(e, f)\right)\right),
$$

and so these two terms exactly cancel. All of the additional terms in $d\left({ }_{I} \bullet J(\gamma)\right)$ can thus be paired in this way to cancel.

Finally, the verification of (iii) is entirely straightforward.

\section{5. $\mathcal{P} \mathcal{G}_{n}$ IS A RESOLUTION OF $H^{*}\left(f \underline{\mathcal{M}}_{n}\right)$}

Kontsevich showed that the map $\mathcal{K G}_{n-1} \rightarrow H^{*}\left(D_{2}(n-1)\right)$, defined by sending a graph with no internal vertices to the corresponding product of Arnold classes $\omega_{i j}$, one for each edge $e_{i j}$, and sending all graphs with internal vertices to zero, is a quasi-isomorphism of cooperads; see [15, Section 3.3.4] or [17, Theorem 9.1]. It 
follows immediately that the analogous map $\mathcal{A G}_{n-1} \rightarrow H^{*}\left(f \underline{\mathcal{M}}_{n}\right)$ is also a quasiisomorphism of cooperads. The cohomology $H^{*}(f \underline{\mathcal{M}})$ is a cyclic cooperad, but $\mathcal{A G}$ is not a cyclic cooperad. Hence we must pass from affine to projective graphs.

Theorem 5.1. The map $\mathcal{P} \mathcal{G}_{V} \rightarrow H^{*}\left(f \underline{\mathcal{M}}_{V}\right)$ defined by sending $\gamma$ to zero if it has any internal vertices, and otherwise sending it to the corresponding product of $\alpha_{i j}$ classes, one for each edge $e_{i j}$, is a quasi-isomorphism of cyclic cooperads.

We will derive this theorem from the corresponding statement for $\mathcal{A G}_{n-1}$ by a spectral sequence comparison of $\mathcal{A G}_{n-1}$ and $\mathcal{P} \mathcal{G}_{n}$. The first step is to construct a map between $\mathcal{A G}_{n-1}$ and $\mathcal{P} \mathcal{G}_{n}$, and for this we must introduce a couple of auxiliary graded vector spaces. Let $\widetilde{\mathcal{A G}}_{n-1}$ denote the graded vector space covering $\mathcal{A G}_{n-1}$ in which each graph is equipped with an injective map from the set of its internal vertices to $\mathbb{N}$. Let $\widetilde{\mathcal{P G}}$, denote the graded vector space covering $\mathcal{P} \mathcal{G}_{n}$ in which the pinwheel relation is not imposed and each graph is equipped with an injective map from the set of its internal vertices to $\mathbb{N}$.

There is an injective linear map

$$
\widetilde{\psi}: \widetilde{\mathcal{A G}}_{n-1} \hookrightarrow \widetilde{\mathcal{P G}}_{n}
$$

defined by sending $\gamma \otimes\left(\eta_{i_{1}} \wedge \cdots \wedge \eta_{i_{k}}\right)$ to the graph constructed by adding a disjoint external vertex 0 , denoted $u_{0}$, and then successively adding an external edge $h_{j}$ between $u_{0}$ and the external vertex labelled by $i_{j}$ for $j=1, \ldots, k$, and then finally by adding a boundary edge $h_{v}$ from $u_{0}$ to each internal vertex $v$. The affine orientation $x$ of $\gamma$ determines a projective orientation of $\widetilde{\psi}\left(\gamma \otimes\left(\eta_{i_{1}} \wedge \cdots \wedge \eta_{i_{k}}\right)\right)$ by the expression

$$
x \wedge h_{1} \wedge \cdots \wedge h_{k} \wedge\left(h_{v} \wedge v\right)_{v \in V_{i n t}},
$$

where $v$ runs over the internal vertices in some arbitrary order.

The image of $\widetilde{\psi}$ is precisely the vector space spanned by graphs for which each internal vertex is connected to $u_{0}$ by an edge. One checks that $\widetilde{\psi}$ descends to a linear map

$$
\psi: \mathcal{A G}_{n-1} \rightarrow \mathcal{P} \mathcal{G}_{n} .
$$

This is a morphism of graded algebras, but it does not commute with the differentials. However, we have the following.

Lemma 5.2. The map $\psi: \mathcal{A G}_{n-1} \rightarrow \mathcal{P} \mathcal{G}_{n}$ is a linear isomorphism.

The idea of the proof of the above lemma is to explicitly construct an inverse to $\psi$ by using the pinwheel relation to rewrite any projective graph as a sum of projective graphs in which each internal vertex is connected by an edge to the external vertex $u_{0}$. We now formalise this procedure. For any $k \in \mathbb{N}$, we define two linear operators

$$
P_{k}, Q_{k}: \widetilde{\mathcal{P G}}_{n} \rightarrow \widetilde{\mathcal{P G}}_{n}
$$

by specifying what they do on generators. Given a projective graph $\gamma \in \widetilde{\mathcal{P G}}_{n}$, if there is no internal vertex labelled by $k$, then $Q_{k}(\gamma)=\gamma$; if there is such an internal vertex, then $Q_{k}$ simply adds an edge $e$ from that vertex to $u_{0}$ or outputs zero if there is already such an edge; the new projective orientation is given by $x \mapsto e \wedge x$. Observe that the operators $Q_{k}$ anticommute.

Let $E(k)$ denote the set of edges in $\gamma$ which are incident at the internal vertex with label $k$, where $E(k)=\emptyset$ if there is no such vertex. 
If $E(k)$ contains an edge to the external vertex $u_{0}$, then $P_{k}(\gamma)=\gamma$; otherwise

$$
P_{k}(\gamma)=-\sum_{e \in E(k)} Q_{k}(\gamma) \backslash e
$$

One can think of $P_{k}$ as using the pinwheel relation to write $\gamma$ as a sum of graphs that are in the image of $\psi$, although we have not yet passed from $\widetilde{\mathcal{P G}}$ to $\mathcal{P G}$.

We can now proceed to define an inverse to $\psi$. Consider the infinite composition

$$
\widetilde{P}_{\infty}:=\left(\cdots \circ P_{2} \circ P_{1}\right): \widetilde{\mathcal{P G}}_{n} \rightarrow \widetilde{\mathcal{P G}}_{n} ;
$$

since any graph $\gamma$ has only finitely many internal vertices, all but finitely many of the $P_{k}$ act by identity on $\gamma$, and so this is well-defined.

Lemma 5.3. $\quad$ (i) $\widetilde{P}_{\infty}$ is equivariant with respect to the action of the group $\Sigma_{\mathbb{N}}$ of permutations of $\mathbb{N}$ by relabelling the internal vertices.

(ii) $\gamma$ and $P_{k}(\gamma)$ project to the same element of $\mathcal{P} \mathcal{G}_{n}$.

(iii) Any two operators $P_{h}, P_{k}$ commute.

(iv) The pinwheel ideal is contained in the kernel of $\widetilde{P}_{\infty}$.

Proof. Part (i) is trivial. Part (ii) is immediate from the pinwheel relation. For part (iii) we need only consider the case where $\gamma$ is a graph with internal vertices $j$ and $k$, neither of which has an edge to $u_{0}$ since in all other cases at least one of $P_{j}$ or $P_{k}$ acts by identity on $\gamma$. In this situation,

$$
P_{j} \circ P_{k}(\gamma)=\sum_{e \in E(k)} \sum_{f \in E(j), f \neq e} Q_{j}\left(Q_{k}(\gamma) \backslash e\right) \backslash f .
$$

The operations of deleting edges $e$ and $f$ anticommute, deleting either $e$ or $f$ anticommutes with $Q_{j}$ and $Q_{k}$, and $Q_{j}$ and $Q_{k}$ anticommute with one another. Hence

$$
Q_{j}\left(Q_{k}(\gamma) \backslash e\right) \backslash f=Q_{j}\left(Q_{k}(\gamma) \backslash f\right) \backslash e .
$$

We turn to part (iv). It suffices to check that for any graph $\gamma$ with internal vertex $k$,

$$
\widetilde{P}_{\infty}\left(\sum_{e \in E(k)} \gamma \backslash e\right)=0 .
$$

Since the operators $P_{k}$ all commute, we need only show that $P_{k}$ sends $\sum_{e \in E(k)}(\gamma \backslash e)$ to zero. First suppose that there is no edge in $\gamma$ between the vertices $k$ and $u_{0}$. Then

$$
\sum_{e \in E(k)} P_{k}(\gamma \backslash e)=-\sum_{e, f \in E(k), f \neq e} Q_{k}(\gamma \backslash e) \backslash f
$$

and this is zero since the $(e, f)$ term cancels exactly with the $(f, e)$ term. Now suppose that there is an edge $h$ in $\gamma$ between the internal vertex labelled $k$ and the external vertex $u_{0}$. Then

$$
\begin{aligned}
P_{k}\left(\sum_{e \in E(k)} \gamma \backslash e\right) & =\sum_{e \in E(k), e \neq h}(\gamma \backslash e)+P_{k}(\gamma \backslash h) \\
& =\sum_{e \in E(k), e \neq h}(\gamma \backslash e)-\sum_{f \in E(k), f \neq h} Q_{k}(\gamma \backslash h) \backslash f=0
\end{aligned}
$$

since $Q_{k}(\gamma \backslash h)=\gamma$, so all terms cancel. 
Proof of Lemma [5.2, Observe that $\widetilde{P}_{\infty}(\gamma)$ lies in the image of $\widetilde{\psi}$. Since $\widetilde{\psi}$ is injective we can thus regard $\widetilde{P}_{\infty}$ as a linear map

$$
\widetilde{P}_{\infty}: \widetilde{\mathcal{P G}}_{n} \rightarrow \widetilde{\mathcal{A G}}_{n-1}
$$

which clearly satisfies $\widetilde{P}_{\infty} \circ \widetilde{\psi}=\mathrm{id}{\widetilde{\mathcal{A G}}_{n-1}}$ and, by Lemma [5.3, descends to a map

$$
P_{\infty}: \mathcal{P} \mathcal{G}_{n} \rightarrow \mathcal{A G}_{n-1}
$$

such that $P_{\infty} \circ \psi=\operatorname{id}_{\mathcal{A G}}$. On the other hand, Lemma 5.3 implies that $\widetilde{P}_{\infty}$ descends to the identity on $\mathcal{P} \mathcal{G}_{n}$. But the induced map on $\mathcal{P} \mathcal{G}_{n}$ is equal to $\psi \circ P_{\infty}$. Therefore $\psi$ is bijective.

The complex $\mathcal{P} \mathcal{G}_{n}$ has a descending filtration

$$
\mathcal{P} \mathcal{G}_{n}=F_{0}\left(\mathcal{P G}_{n}\right) \supset F_{1}\left(\mathcal{P G}_{n}\right) \supset \cdots \supset F_{n-1}(\mathcal{P G}) \supset F_{n}\left(\mathcal{P G}_{n}\right)=0
$$

in which the $k$-th filtration level, $F_{k}\left(\mathcal{P} \mathcal{G}_{n}\right) \subset \mathcal{P} \mathcal{G}_{n}$, is the subcomplex spanned by projective graphs having at least $k$ edges connecting $u_{0}$ to other external vertices. The filtration is well-defined because the pinwheel relation does not affect edges between external vertices, and similarly, the differential is compatible with the filtration. There is a spectral sequence $E_{r}\left(\mathcal{P} \mathcal{G}_{n}\right)$ associated with this filtration.

Lemma 5.4. The map $\psi$ induces an isomorphism of complexes $\mathcal{A G}_{n-1} \cong E_{0}\left(\mathcal{P} \mathcal{G}_{n}\right)$.

Proof. Let us filter $\mathcal{A G}_{n-1}=\mathcal{K G}_{n-1} \otimes \Lambda\left(\eta_{1}, \ldots, \eta_{n-1}\right)$ so that $F_{k}\left(\mathcal{A G}_{n-1}\right)$ is spanned by elements $\gamma \otimes \omega$, where $\omega$ is a product of at least $k$ distinct generators $\eta_{i}$. The linear isomorphism $\psi: \mathcal{A G}_{n-1} \rightarrow \mathcal{P} \mathcal{G}_{n}$ respects the filtrations. We show that if $\gamma \otimes \omega \in F_{k}\left(\mathcal{A G}_{n-1}\right)$, then

$$
d \psi(\gamma \otimes \omega)=\psi(d \gamma \otimes \omega)+\epsilon(\gamma \otimes \omega)
$$

where the error term, $\epsilon(\gamma \otimes \omega)$, is in strictly higher filtration, i.e. $\epsilon(\gamma \otimes \omega) \epsilon$ $F_{k+1}\left(\mathcal{P} \mathcal{G}_{n}\right)$. This will prove the lemma. Recall that $\psi(\gamma \otimes \omega)$ has an edge from the external vertex $u_{0}$ to each internal vertex $v$; let $h_{v}$ denote this edge. The differential of $\psi(\gamma \otimes \omega)$ is a sum of terms of the form $\psi(\gamma \otimes \omega) \oslash(e, f)$; we now consider the various possible positions of the pair of adjacent edges $(e, f)$ in $\psi(\gamma \otimes \omega)$.

Case 1: $e$ is an internal edge from $v_{1}$ to $v_{2}$. If $f$ connects $v_{2}$ to the external vertex $u_{0}$, then $\psi(\gamma \otimes \omega) \oslash(e, f)=\psi(\gamma / e \otimes \omega)$. To see that the orientations agree, suppose $\gamma$ has affine orientation $x$. Then $\psi(\gamma \otimes \omega)$ has the projective orientation

$$
x \wedge(\omega) \wedge\left(h_{v} \wedge v\right)_{v \in V_{i n t}}=x \wedge(\omega) \wedge f \wedge v_{2} \wedge\left(h_{v} \wedge v\right)_{v \neq v_{2}},
$$

where $(\omega)$ here stands for a wedge product of the boundary edges incident at $u_{0}$ corresponding to the class $\omega$, and $\psi(\gamma \otimes \omega) \oslash(e, f)$ has projective orientation

$$
\iota_{e} x \wedge(\omega) \wedge\left(h_{v} \wedge v\right)_{v \neq v_{2}},
$$

which is precisely the projective orientation of $\psi(\gamma / e \otimes \omega)$. If $f$ connects $v_{2}$ to a vertex that is not $u_{0}$, then $\psi(\gamma) \oslash(e, f)=0$ because this graph has a double edge coming from the edges $u_{0} v_{1}$ and $u_{0} v_{2}$ in $\psi(\gamma)$.

Case 2: $e$ goes from an external vertex $x \neq u_{0}$ to an internal vertex $v$. If $f=h_{v}$ is the edge from $u_{0}$ to $v$, then $\psi(\gamma \otimes \omega) \oslash(e, f)=\psi(\gamma / e \otimes \omega)$ as in case 1 . The remaining possibilities for the position of $f$ will contribute only to the error term $\epsilon(\gamma \otimes \omega)$. If $f \neq h_{v}$, then $\psi(\gamma \otimes \omega) \oslash(e, f)$ is in strictly higher filtration because it has an additional external edge between $x$ and $u_{0}$. 
Case 3: $e$ goes from $u_{0}$ to an internal vertex $v$. The internal vertices are at least 4 -valent, so there are at least 3 edges connecting $v$ to vertices $a, b, c$ other than $u_{0}$. We may assume that $f$ is not the edge from $v$ to $a$. If $a$ is internal, then $\psi(\gamma \otimes \omega) \oslash(e, f)=0$ because it has a double edge coming from the edges from $a$ to $v$ and from $a$ to $u_{0}$ in $\psi(\gamma \otimes \omega)$. If $a$ is external, then $\psi(\gamma \otimes \omega) \oslash(e, f)$ has one more edge than $\psi(\gamma \otimes \omega)$ connecting $a$ to $u_{0}$ and thus lives in a higher filtration.

Proof of Theorem 5.1. By Lemma 5.4 the $E_{1}$-term is $E_{1}\left(\mathcal{P} \mathcal{G}_{n}\right) \cong H^{*}\left(\mathcal{A G}_{n-1}\right)$. On the other hand, by definition of the affine graph complex (Definition 4.3), and since Kontsevich's graph complex $\mathcal{K G}_{n-1}$ is a resolution of $H^{*}\left(D_{2}(n-1)\right)$,

$$
\begin{aligned}
H^{*}\left(\mathcal{A G}_{n-1}\right) & \cong H^{*}\left(\mathcal{K G}_{n-1}\right) \otimes \Lambda\left(\eta_{1}, \ldots, \eta_{n-1}\right) \\
& \cong H^{*}\left(D_{2}(n-1)\right) \otimes \Lambda\left(\eta_{1}, \ldots, \eta_{n-1}\right) \\
& \cong H^{*}\left(f \underline{\mathcal{M}}_{n}\right) .
\end{aligned}
$$

We claim that the spectral sequence collapses at the $E_{1}$-term. The projection $\mathcal{P} \mathcal{G}_{n} \rightarrow H^{*}\left(f \underline{\mathcal{M}}_{n}\right)$ is surjective in cohomology because the forms $\alpha_{i j}$ generate the cohomology ring of $f \mathcal{M}_{n}$ and $\alpha_{i j}$ is the image of the graph with a single edge between external vertices labelled $i$ and $j$. Thus the dimension of $H^{*}\left(\mathcal{P} \mathcal{G}_{n}\right)$ is not smaller than the dimension of $H^{*}\left(f \underline{\mathcal{M}}_{n}\right)$. The isomorphism $E_{1}\left(\mathcal{P} \mathcal{G}_{n}\right) \cong H^{*}\left(f \underline{\mathcal{M}}_{n}\right)$ and finite dimensionality imply that $E_{1}\left(\mathcal{P} \mathcal{G}_{n}\right) \cong E_{\infty}\left(\mathcal{P} \mathcal{G}_{n}\right)$ and $q^{*}: H^{*}\left(\mathcal{P} \mathcal{G}_{n}\right) \stackrel{\cong}{\rightrightarrows}$ $H^{*}\left(f \underline{\mathcal{M}}_{n}\right)$ is an isomorphism of cyclic cooperads.

\section{The Kontsevich InTEGRAL FROM GRAPHS TO FORMS}

In this section we construct a quasi-isomorphism

$$
\mathscr{I}: \mathcal{P} \mathcal{G}_{V} \rightarrow \Omega_{P A}^{*}\left(f \underline{\mathcal{M}}_{V}\right)
$$

where $\Omega_{P A}^{*}$ is the complex of "piecewise semi-algebraic forms". The idea is as follows. A graph $\gamma=\left(V, V_{i n t}, E\right)$ determines a differential form on $f \underline{\mathcal{M}}_{V \sqcup V_{\text {int }}}$ given by multiplying $\alpha_{i j}$ 1-forms corresponding to the edges, and one then integrates this form along the fibres of the bundle $f \underline{\mathcal{M}}_{V \sqcup V \text { int }} \rightarrow f \underline{\mathcal{M}}_{V}$ to obtain a form on $f \underline{\mathcal{M}}_{V}$. Both the integrand and the integration map have a sign ambiguity, but if $\gamma$ has a projective orientation, then these sign ambiguities are resolved. The differential form that results is generally not smooth, and so we must pass to the semi-algebraic setting in which the Kontsevich integral is well-defined.

6.1. PA forms and fibrewise integration. Given a semi-algebraic manifold, the complex of minimal forms, $\Omega_{\text {min }}^{*}(X)$, is spanned by elements

$$
f_{0} \cdot d f_{1} \wedge \cdots \wedge d f_{k}
$$

where the $f_{i}$ are semi-algebraic functions. The complex of $P A$ forms, $\Omega_{P A}^{*}(X)$, consists of those forms which can be written as a pushforward of a minimal form. When $X$ is smooth, the commutative differential graded algebra of $P A$ forms is quasi-isomorphic to the ordinary smooth forms.

We first recall some facts about semi-algebraic fibrewise integration. Suppose $\pi: E \rightarrow B$ is a semi-algebraic fibre bundle with $n$-dimensional compact fibres and equipped with a fibrewise orientation. Integration of forms fibrewise defines a map (see [11, Section 8])

$$
\pi_{!}: \Omega_{\min }^{*}(E) \rightarrow \Omega_{P A}^{*-n}(B) .
$$


In particular, if $\omega \in \Omega_{m i n}^{n}(E)$ restricts to a volume form on each fibre, then $\pi_{!} \omega$ is the semi-algebraic function on $B$ which sends $b \in B$ to the volume of the fibre over $b$.

We shall need iterated fibrewise integrals at several points in this section. As the theory is developed in [11], the pushforward of a minimal form is a $P A$ form by definition, and the pushforward of a $P A$ form is not defined. Note that the minimal forms constitute a sub-CDGA of all $P A$ forms.

Proposition 6.1. If $p: E \rightarrow B$ and $q: B \rightarrow X$ are semi-algebraic bundles with compact oriented fibres, and $\xi$ is a minimal form on $E$ such that $p_{!} \xi$ is a minimal form on $B$, then $q_{!}\left(p_{!} \xi\right)$ is defined and

$$
q_{!}\left(p_{!} \xi\right)=(q \circ p) ! \xi
$$

This is a slight generalisation of [11, Prop 8.11] and has a similar proof.

Given any form $\xi \in \Omega_{m i n}^{*}(B)$, one has the push-pull formula ([11, Prop. 8.13]),

$$
\pi_{!}\left(\pi^{*} \xi \cdot \omega\right)=\xi \cdot \pi ! \omega .
$$

Now recall the fibrewise Stokes formula.

Theorem 6.2 ([11, Prop. 8.10]). Let $\pi: E \rightarrow B$ be a semi-algebraic bundle of oriented $k$-manifolds with boundary, with $\left.\pi\right|_{\partial}: \partial E \rightarrow B$ denoting the associated fibrewise boundary bundle. Then, giving the boundary the induced orientation,

$$
d\left(\pi_{!} \xi\right)=\pi_{!}(d \xi)+(-1)^{\operatorname{deg} \xi-k}\left(\left.\pi\right|_{\partial}\right) ! \xi,
$$

for any form $\xi \in \Omega_{\text {min }}^{*}(E)$.

6.2. Definition of the integration map $\mathscr{I}$. We first define $\mathscr{I}$ on the subcomplex $\mathcal{P G}_{V}^{0}$ of graphs with no internal vertices. Let $\gamma \in \mathcal{P} \mathcal{G}_{V}^{0}$ be such a graph with edge set $E$ and orientation ray in $\operatorname{det}(E)$ spanned by $e_{1} \wedge \cdots \wedge e_{k}$. An edge $e$ between vertices $u$ and $v$ determines a semi-algebraic minimal 1 -form

$$
\alpha_{e}:=\alpha_{u v}=\pi_{u v}^{*} d \theta \in \Omega_{\text {min }}^{1}\left(f \underline{\mathcal{M}}_{V}\right),
$$

where $\pi_{u v}: f \underline{\mathcal{M}}_{V} \rightarrow f \underline{\mathcal{M}}_{\{u, v\}} \cong S^{1}$ forgets all marked points except $u$ and $v$. Define

$$
\mathscr{I}(\gamma):=\alpha_{e_{1}} \wedge \cdots \wedge \alpha_{e_{k}} \in \Omega_{m i n}^{*}\left(f \underline{\mathcal{M}}_{V}\right) .
$$

Recall that (the equivalence class of) a projectively oriented graph $\gamma$ with internal vertex set $V_{\text {int }}$ and external vertex set $V$ can be written as $\pi_{r}^{V_{\text {int }}}(\widetilde{\gamma}) \in \mathcal{P} \mathcal{G}_{V}$ for some ray $r \subset \operatorname{det}\left(V_{\text {int }}\right)$ and graph $\widetilde{\gamma} \in \mathcal{P} \mathcal{G}_{V \sqcup V_{\text {int }}}^{0}$. We then define

$$
\mathscr{I}(\gamma)=\mathscr{I}\left(\pi_{r}^{V_{i n t}}(\widetilde{\gamma})\right):=\frac{1}{2^{\left|V_{i n t}\right|}} \pi_{!}^{V_{i n t}} \mathscr{I}(\widetilde{\gamma}),
$$

where the fibrewise orientation of $\pi^{V_{\text {int }}}: f \underline{\mathcal{M}}_{V \sqcup V_{\text {int }}} \rightarrow f \underline{\mathcal{M}}_{V}$ is determined by the ray $r$ as described in section 2.4. We prove in Lemma 6.3 below that $\mathscr{I}$ respects the relations $(R 1-4)$ of Definition 4.4, and hence it descends to a map from $\mathcal{P} \mathcal{G}_{V}$, which we denote by the same name.

Lemma 6.3. The Kontsevich integral satisfies:

(i) $\mathscr{I}(\gamma)=-\mathscr{I}(\bar{\gamma})$.

(ii) $\mathscr{I}$ vanishes on graphs having an internal vertex of valence $\leq 3$.

(iii) $\mathscr{I}$ vanishes on graphs which have a connected component containing at most one vertex in $V$ and at least one internal vertex.

(iv) The pinwheel ideal is contained in the kernel of $\mathscr{I}$. 
Hence we have a well-defined morphism $\mathscr{I}: \mathcal{P} \mathcal{G}_{V} \rightarrow \Omega_{P A}^{*}\left(f \underline{\mathcal{M}}_{V}\right)$ of graded algebras.

Proof. Statement (i) is trivial. For (ii), suppose $\gamma$ has a trivalent internal vertex $x$ adjacent to vertices $u, v$ and $w$ as below.

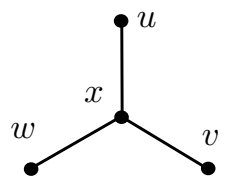

By Proposition 6.1 one may integrate out the internal vertices by first integrating out $x$ and then integrating out the remaining internal vertices, i.e.

$$
\pi_{!}^{V_{i n t}} \mathscr{I}(\widetilde{\gamma})=(-1)^{\left|V_{i n t}\right|-1} \pi_{!}^{V_{i n t} \backslash\{x\}} \pi_{!}^{\{x\}} \mathscr{I}(\widetilde{\gamma})
$$

where if $\pi^{V_{\text {int }}}$ has fibrewise orientation $r \subset \operatorname{det}\left(V_{\text {int }}\right)$, then $\pi^{V_{\text {int }} \backslash\{x\}}$ has fibrewise orientation $\iota_{x} r \subset \operatorname{det}\left(V_{\text {int }} \backslash\{x\}\right)$ and $\pi^{\{x\}}$ has its standard fibrewise orientation. This works because the integrand $\mathscr{I}(\widetilde{\gamma})$ is the product of $\alpha_{u x} \alpha_{v x} \alpha_{w x}$ with a minimal form $\beta$ that is independent of $x$, so by formula (6.1.1), $\left(\pi^{\{x\}}\right) ! \mathscr{I}(\widetilde{\gamma})$ is equal to the product of $\beta$ with the $P A$ form

$$
\pi_{!}^{\{x\}}\left(\alpha_{u x} \alpha_{v x} \alpha_{w x}\right)
$$

and since the fibres of $\pi^{\{x\}}: f \underline{\mathcal{M}}_{V \sqcup V_{i n t}} \rightarrow f \underline{\mathcal{M}}_{V \sqcup V_{i n t} \backslash\{x\}}$ have dimension 3, (6.2.1) is a semi-algebraic function on $f \underline{\mathcal{M}}_{V \sqcup V_{i n t} \backslash\{x\}}$, and so $\left(\pi^{\{x\}}\right) ! \mathscr{I}(\widetilde{\gamma})$ is minimal. Putting $u, v$ and $w$ at 0,1 and $\infty$ identifies each fibre with the complement of a codimension 1 semi-algebraic subset of the unit tangent circle bundle of the Riemann sphere punctured at 0,1 and $\infty, S\left(T\left(S^{2} \backslash\{0,1, \infty\}\right)\right)$, and the differential form $\alpha_{u x} \alpha_{v x} \alpha_{w x}$ extends to $S\left(T\left(S^{2} \backslash\{0,1, \infty\}\right)\right)$. Complex conjugation is an orientation-preserving diffeomorphism of the tangent circle bundle, and it reverses the sign of $\alpha_{u x} \alpha_{v x} \alpha_{w x}$ since it reverses the sign of each of the three factors. Hence the integral over each fibre is zero, and the fibrewise integral (6.2.1) is identically zero.

Statement (iii) follows from Kontsevich's lemma on the vanishing of integrals over configuration spaces [16, Lemma 6.4]. We choose an external vertex as the point at infinity, and write a form $\mathscr{I}(\widetilde{\gamma})$ as a polynomial in the forms $\omega_{i j}$ and $\eta_{k}$. Integrate out the internal vertices by first integrating out the tangent ray decorations at the internal vertices, resulting in a $P A$ and in fact minimal form on a configuration space; what remains is a configuration space integral of the form that Kontsevich's lemma refers to.

For (iv), we will show that if $v$ is an internal vertex, then integrating out the tangent ray decoration at $v$ sends the element

$$
\sum_{e \in E(v)} \mathscr{I}(\widetilde{\gamma} \backslash e)
$$


to zero. Write the orientation of $\widetilde{\gamma}$ as $\beta \wedge e_{0} \wedge \cdots \wedge e_{k}$, where $E(v)=\left\{e_{0}, \ldots, e_{k}\right\}$ is the set of edges incident at $v$. Let $u_{k}$ denote the opposite endpoint of $e_{k}$. Then

$$
\begin{aligned}
& \sum_{e \in E(v)} \widetilde{\gamma} \backslash e=\beta \sum_{i=0}^{k}(-1)^{i} \alpha_{e_{0}} \alpha_{e_{1}} \cdots \widehat{\alpha_{e_{i}}} \cdots \alpha_{e_{k}} \\
& \quad=\beta\left(\eta_{v}+\eta_{u_{1}}-2 \omega_{v u_{1}}\right) \cdots\left(\eta_{v}+\eta_{u_{k}}-2 \omega_{v u_{k}}\right) \\
& \quad+\beta \eta_{v} \sum_{i=1}^{k}(-1)^{i}\left(\eta_{v}+\eta_{u_{1}}-2 \omega_{v u_{1}}\right) \cdots\left(\eta_{v}+\widehat{\eta_{u_{i}}-2} \omega_{v u_{i}}\right) \cdots\left(\eta_{v}+\eta_{u_{k}}-2 \omega_{v u_{k}}\right),
\end{aligned}
$$

and integrating out the decoration at $v$ extracts the coefficient of $\eta_{v}$. It is not hard to see that the coefficient of $\eta_{v}$ is in fact zero.

6.3. Differentials of graph forms. Here we shall prove the following.

Theorem 6.4. The linear map $\mathscr{I}: \mathcal{P} \mathcal{G}_{V} \rightarrow \Omega_{P A}^{*}\left(f \underline{\mathcal{M}}_{V}\right)$ commutes with the differentials and is thus a morphism of differential graded algebras.

Let $\gamma \in \mathcal{P} \mathcal{G}_{V}$ be a projectively oriented graph. We will prove this theorem by using the Stokes formula to compute the exterior derivative of the PA form $\mathscr{I}(\gamma)$. Write $\gamma=\pi_{r}^{V_{\text {int }}}(\widetilde{\gamma})$. By the fibrewise Stokes formula (Theorem 6.2),

$$
d \mathscr{I}(\gamma)=d\left(\frac{1}{2^{\left|V_{i n t}\right|}} \pi_{!}^{V_{i n t}} \mathscr{I}(\widetilde{\gamma})\right)=\frac{(-1)^{|E|+\left|V_{i n t}\right|}}{2^{\left|V_{i n t}\right|}} \sum_{U_{1}, U_{2}}\left(\left.\pi^{V_{i n t}}\right|_{\partial_{U_{1}, U_{2}}}\right), \mathscr{I}(\widetilde{\gamma}),
$$

since $\mathscr{I}(\widetilde{\gamma})$ is closed. We can factor $\left.\pi^{V_{i n t}}\right|_{\partial_{U_{1}, U_{2}}}$ into the composition of first forgetting the points in $U_{1} \cap V_{\text {int }}$ and then forgetting the remaining internal vertices:

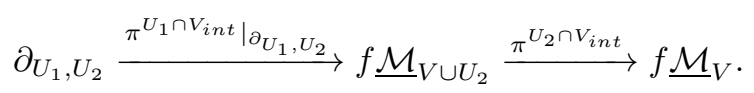

By Lemma 2.4, the fibrewise boundary component $\partial_{U_{1}, U_{2}}$ is the (closure of the) locus where a node separates the sphere into two lobes $S_{1}, S_{2}$, with the points labelled by $U_{i}$ on the lobe $S_{i}$. The map $\left.\pi^{U_{1} \cap V_{i n t}}\right|_{\partial_{U_{1}, U_{2}}}$ forgets the first lobe if $U_{1} \cap V=\emptyset$, and collapses it to a single marked point if $U_{1}$ contains an external vertex; hence it is a semi-algebraic fibre bundle, and in fact it is trivial.

Lemma 6.5. If $\left|U_{1}\right| \geq 3$, then the corresponding term in the Stokes formula vanishes:

$$
\left(\left.\pi^{V_{\text {int }}}\right|_{\partial_{U_{1}, U_{2}}}\right) ! \mathscr{I}(\widetilde{\gamma})=0 .
$$

Proof. Consider performing the fibrewise integration by first integrating along the fibres of $\left.\pi^{U_{1} \cap V_{i n t}}\right|_{\partial_{U_{1}, U_{2}}}$ and then integrating out the remaining internal vertices. Furthermore, consider performing the integral over the fibres of $\left.\pi^{U_{1} \cap V_{i n t}}\right|_{\partial_{U_{1}, U_{2}}}$ by first integrating out the tangent ray decorations of the points in $U_{1} \cap V_{\text {int }}$ and then integrating out the positions of these points:

$$
\left(\left.\pi^{U_{1} \cap V_{\text {int }}}\right|_{\partial_{U_{1}, U_{2}}}\right) !=\left(\pi_{\mathrm{pos}}\right) ! \circ\left(\pi_{\mathrm{dec}}\right) ! \cdot
$$

Since iterated integration of $P A$ forms is not defined in general, we must justify as above both of these iterated integrations. We will see that each successive integration results again in a minimal form, so the iteration is allowed.

Relative to a choice of a point $x \in U_{2}$ as a point at infinity, the form $\mathscr{I}(\widetilde{\gamma})$ can be expanded in terms of the affine presentation generators as in the proof of Lemma 
6.3 (iii),(iv). Integrating out the $V_{\text {int }} \cap U_{1}$ decorations then yields a polynomial $P=\left(\pi_{\mathrm{dec}}\right) ! \mathscr{I}(\widetilde{\gamma})$ in $\omega_{i j}$ forms and $\eta_{k}$ forms for $k \notin V_{i n t} \cap U_{1}$, and hence a minimal form. On the locus $\partial_{U_{1}, U_{2}}$, let $q$ denote the node between the two lobes. On this locus, if $a \in U_{1}$ and $b \in U_{2}$, then $\omega_{a b}=\omega_{q b}$. Hence each monomial in $P$ factors as a product $\beta_{2} \wedge \beta_{1}$, where $\beta_{2}$ is a monomial of $\omega$ and $\eta$ forms on the second lobe (relative to $x$ and including $q$ as a marked point) and $\beta_{1}$ is a monomial of only $\omega$ forms on the first lobe (relative to $q$ ). Now, integrating out the $V_{i n t} \cap U_{1}$ positions,

$$
\left(\pi_{\text {pos }}\right) !\left(\beta_{2} \beta_{1}\right)=\beta_{2} \cdot\left(\pi_{\text {pos }}\right) !\left(\beta_{1}\right)
$$

and if $\left|U_{1}\right| \geq 3$, then $\left(\pi_{\text {pos }}\right) !\left(\beta_{1}\right)=0$ by Kontsevich's Vanishing Lemma [16, Lemma $6.4]$.

Thus the only fibrewise boundary components which contribute are those for which $\left|U_{1}\right|=2$. If $U_{1}=\{u, v\}$, then we will write $\partial_{u v}=\partial_{U_{1}, U_{2}}$. We will now compute the Stokes boundary term $\left(\left.\pi^{V_{i n t}}\right|_{\partial_{u v}}\right) ! \mathscr{I}(\widetilde{\gamma})$. Factor $\left.\pi^{V_{i n t}}\right|_{\partial_{u v}}$ as

$$
\partial_{u v} \stackrel{\left.\pi^{\{v\}}\right|_{\partial_{u v}}}{\longrightarrow} f \underline{\mathcal{M}}_{V \sqcup V_{i n t} \backslash\{v\}} \stackrel{\pi^{V_{i n t} \backslash\{v\}}}{\longrightarrow} f \underline{\mathcal{M}}_{V} .
$$

The first map is given its standard fibrewise orientation, and the second map is given the fibrewise orientation $\iota_{x} r$, where $r \subset \operatorname{det}\left(V_{\text {int }}\right)$ is the fibrewise orientation of $\pi^{V_{i n t}}$. Note that with these choices of orientation,

$$
\pi_{!}^{V_{\text {int }} \backslash\{v\}} \circ \pi_{!}^{\{v\}}=(-1)^{\left|V_{i n t}\right|-1} \pi_{!}^{V_{i n t}} .
$$

The Stokes formula (6.3.1) then becomes

$$
d \mathscr{I}(\gamma)=\frac{(-1)^{|E|-1}}{2^{\left|V_{i n t}\right|}} \sum_{\{u, v\}} \pi_{!}^{V_{i n t} \backslash\{v\}}\left(\left.\pi^{\{v\}}\right|_{\partial_{u v}}\right) ! \mathscr{I}(\widetilde{\gamma}),
$$

where the sum runs over unordered pairs $\{u, v\} \subset V \sqcup V_{\text {int }}$ at most one of which is external (if one is internal, then we call it $v$, and if both are internal, then we choose one to call $v$ ).

Lemma 6.6. Suppose we are given four distinct vertices $u, v, w, x$. Then on $\partial_{u v}$,

$$
\alpha_{u w} \alpha_{u x}=(1 / 2)\left(\alpha_{v w}-\alpha_{v x}\right)\left(\alpha_{u w}+\alpha_{u x}\right) .
$$

Proof. We can consider $x$ to be at infinity and in the corresponding affine presentation, $\omega_{u w}=\omega_{v w}$. Hence, on $\partial_{u v}$ we have the relation $\alpha_{u x}-\alpha_{u w}=\alpha_{v x}-\alpha_{v w}$. The result follows from multiplying both sides with $\alpha_{v x}+\alpha_{v w}$ since 1-forms anticommute.

Lemma 6.7. For any triple of vertices $(u, v, w)$, the 2 -form

$$
(1 / 2) \alpha_{w v} \alpha_{v u}
$$

is a fibrewise negative unit volume form for $\left.\pi^{\{v\}}\right|_{\partial_{u v}}: \partial_{u v} \rightarrow f \underline{\mathcal{M}}_{V \sqcup V_{\text {int }} \backslash\{v\}}$.

Proof. In terms of the affine presentation (relative to $w$ ), one sees that $\omega_{u v} \eta_{v}$ is a fibrewise negative unit volume form for $\left.\pi^{\{v\}}\right|_{\partial_{u v}}: \partial_{u v} \rightarrow f \underline{\mathcal{M}}_{V \sqcup V_{i n t} \backslash\{v\}}$ as follows. We factor $\pi^{\{v\}}$ as $\pi_{\text {pos }}^{\{v\}} \circ \pi_{\text {dec }}^{\{v\}}$, and each of these maps has a fibrewise orientation as described in section 2.4. The form $\eta_{v}$ is a fibrewise positive unit volume form for $\pi_{\mathrm{dec}}^{\{v\}}$. The form $\omega_{u v}$ is a negative fibrewise unit volume form for the restriction 
of $\pi_{\text {pos }}^{\{v\}}$ to the face $\pi_{\text {dec }}^{\{v\}}\left(\partial_{u v}\right)=\partial_{u v} / S^{1} \subset f \underline{\mathcal{M}}_{V \sqcup V_{i n t}} / S^{1}$. Translating to the cyclic presentation,

$$
\begin{aligned}
\omega_{u v} \eta_{v} & =(1 / 2)\left(\alpha_{w u}+\alpha_{w v}-\alpha_{u v}\right) \alpha_{w v} \\
& =(1 / 2)\left(\alpha_{w u} \alpha_{w v}-\alpha_{u v} \alpha_{w v}\right) .
\end{aligned}
$$

Since $\alpha_{w u}$ is constant on the fibres of $\left.\pi^{\{v\}}\right|_{\partial_{u v}}$, the first term in the final line integrates to zero on each fibre, and so we obtain the result.

Lemma 6.8. Consider a vertex set $W=\{1, \ldots, k, u, v\}$. On the boundary component $\partial_{u v} \subset f \underline{\mathcal{M}}_{W}$, the following identity holds:

$$
\alpha_{1 v} \cdots \alpha_{k v}=(1 / 2)^{k-1}\left(\sum_{i=1}^{k}(-1)^{k+i} \alpha_{1 u} \cdots \widehat{\alpha_{i u}} \cdots \alpha_{k u}\right)\left(\alpha_{1 v}+\sum_{j=2}^{k} 2^{j-2} \alpha_{j v}\right) .
$$

Proof. Use induction on $k$ and the relation (6.6).

Proof of Theorem 6.4. Suppose $\gamma$ is a projective graph. Write $\gamma=\pi_{r}^{V_{\text {int }}}(\widetilde{\gamma})$. Given an edge $e$ between vertices $u, v$ with $v$ internal (if $u$ is internal as well, then we orient $e$ with head at $v$ ) and a second edge $f$ meeting $v$,

$$
\gamma \oslash(e, f)=(-1)^{|E|} \pi_{\iota_{v} r}^{V_{i n t}} \backslash\{v\}(\widetilde{\gamma} \oslash(e, f)),
$$

where the sign comes from sliding $v$ to the left past the edges $E \backslash\{f, e\}$. By Lemmas 6.8 and 6.7.

$$
\left(\left.\pi^{\{v\}}\right|_{\partial_{u v}}\right) ! \mathscr{I}(\widetilde{\gamma})=(-2) \sum_{f \in E(v) \backslash\{e\}} \mathscr{I}(\widetilde{\gamma} \oslash(e, f)) .
$$

By combining these we find that

$$
\begin{aligned}
\sum_{f} \mathscr{I}(\gamma \oslash(e, f)) & =\left(\frac{(-1)^{|E|-1}}{2^{\left|V_{i n t}\right|-1}}\right) \sum_{f} \pi_{!}^{V_{i n t} \backslash\{v\}} \mathscr{I}(\widetilde{\gamma} \oslash(e, f)) \\
& =\left(\frac{(-1)^{|E|-1}}{2^{\left|V_{i n t}\right|}}\right) \pi_{!}^{V_{i n t} \backslash\{v\}}\left(\left.\pi^{\{v\}}\right|_{\partial_{u v}}\right) ! \mathscr{I}(\widetilde{\gamma}) .
\end{aligned}
$$

Summing this over all unordered pairs $\{u, v\}$ and using (6.3.2) then gives the desired result.

6.4. Example: resolving the cyclic Arnold relation. As an illustration of Theorem 6.4. let us consider the product $(1 / 2) \alpha_{v a} \alpha_{v b} \alpha_{v c} \alpha_{v d}$ corresponding to the graph $\gamma$ shown below,

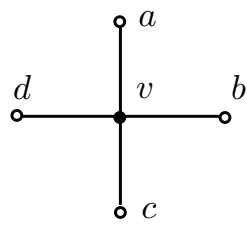


It has internal vertex $v$ and projective orientation $\left(e_{a v} \wedge e_{b v} \wedge e_{c v} \wedge e_{d v} \wedge v\right)$. We can express $\left(\left.\pi^{\{v\}}\right|_{\partial_{d v}}\right) ! \mathscr{I}(\widetilde{\gamma})$ as a linear combination of edge forms as follows. On $\partial_{d v}$,

$$
\begin{aligned}
\alpha_{v a} \alpha_{v b} \alpha_{v c} \alpha_{d v}= & (1 / 2)\left(\alpha_{d a}-\alpha_{d b}\right)\left(\alpha_{v a}+\alpha_{v b}\right) \alpha_{v c} \alpha_{d v} \\
= & (1 / 4)\left(\alpha_{d a}-\alpha_{d b}\right)\left(\alpha_{d a}-\alpha_{d c}\right)\left(\alpha_{v a}+\alpha_{v c}\right) \alpha_{d v} \\
& +(1 / 4)\left(\alpha_{d a}-\alpha_{d b}\right)\left(\alpha_{d b}-\alpha_{d c}\right)\left(\alpha_{v b}+\alpha_{v c}\right) \alpha_{d v} \\
= & (1 / 4)\left(\alpha_{d a} \alpha_{d b}-\alpha_{d a} \alpha_{d c}+\alpha_{d b} \alpha_{d c}\right)\left(\alpha_{v a} \alpha_{d v}+\alpha_{v b} \alpha_{d v}+2 \alpha_{v c} \alpha_{d v}\right) .
\end{aligned}
$$

By Lemma 6.7,

$$
\left(\left.\pi^{\{v\}}\right|_{\partial_{d v}}\right) !\left((1 / 2) \alpha_{v a} \alpha_{v b} \alpha_{v c} \alpha_{v d}\right)=-\left(\alpha_{d a} \alpha_{d b}-\alpha_{d a} \alpha_{d c}+\alpha_{d b} \alpha_{d c}\right) .
$$

This corresponds to the sum of graphs

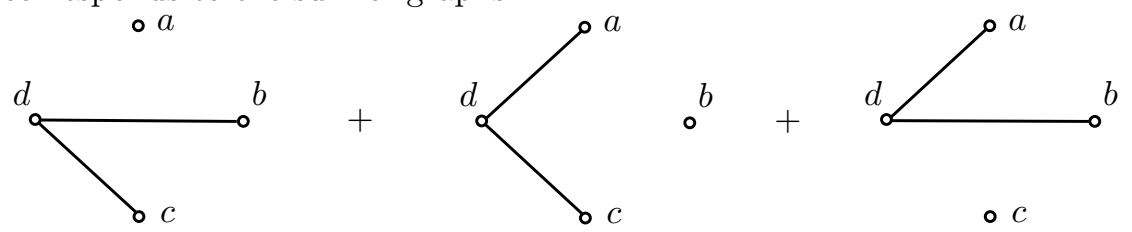

where each is given the orientation induced from that of $\gamma$ by first deleting an edge and then contracting the $\{d, v\}$ edge. From this one finds that

$$
d(\text { plus graph })=(\text { cyclic Arnold relation }) \text {. }
$$

\section{Compatibility of the Kontsevich integral WITH THE CYCLIC OPERAD STRUCTURE}

The $P A$-forms on $f \mathcal{M}$ do not constitute a cyclic cooperad because the exterior product of forms, $\Omega_{P A}^{*}(X) \otimes \Omega_{P A}^{*}(Y) \rightarrow \Omega_{P A}^{*}(X \times Y)$ is invertible up to quasiisomorphism but not on the nose. However, Lemma 7.2 below shows compatibility between the cyclic operad structure on $f \underline{\mathcal{M}}$ and the cyclic cooperad structure on $\mathcal{P G}$.

Lemma 7.1. For $u, v \in(I \backslash\{i\}) \sqcup(J \backslash\{j\})$, the pullback of $\alpha_{u v}$ via ${ }_{i}{ }_{j}: f \underline{\mathcal{M}}_{I} \times$ $f \underline{\mathcal{M}}_{J} \rightarrow f \underline{\mathcal{M}}_{I \sqcup J \backslash\{i, j\}}$ is given by:

$$
\left({ }_{i} \circ_{j}\right)^{*} \alpha_{u v}= \begin{cases}\alpha_{u v} \otimes 1 & \text { if } u, v \in I, \\ 1 \otimes \alpha_{u v} & \text { if } u, v \in J, \\ \alpha_{u i} \otimes 1+1 \otimes \alpha_{j v} & \text { if } u \in I \text { and } v \in J .\end{cases}
$$

Proof. If $u$ and $v$ are both in $I$, then commutativity of the diagram

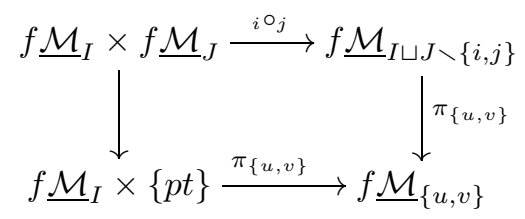

implies the formula in this case; the reasoning is the same when $u, v \in J$. Suppose now that $u \in I \backslash\{i\}$ and $v \in J \backslash\{j\}$. Choosing an identification $f \underline{\mathcal{M}}_{2} \cong S^{1}$, the map

$$
{ }_{i}^{\circ}{ }_{j}: f \underline{\mathcal{M}}_{\{u, i\}} \times f \underline{\mathcal{M}}_{\{j, v\}} \rightarrow f \underline{\mathcal{M}}_{\{u, v\}}
$$


corresponds with the product map $\mu: S^{1} \times S^{1} \rightarrow S^{1}$, and $\mu^{*} d \theta=d \theta \otimes 1+1 \otimes d \theta$. Commutativity of the diagram

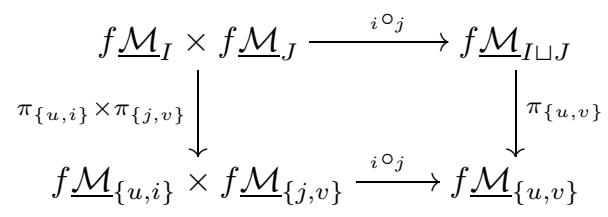

then implies the formula.

Lemma 7.2 ([17, Proposition 8.19]). The following diagram commutes:

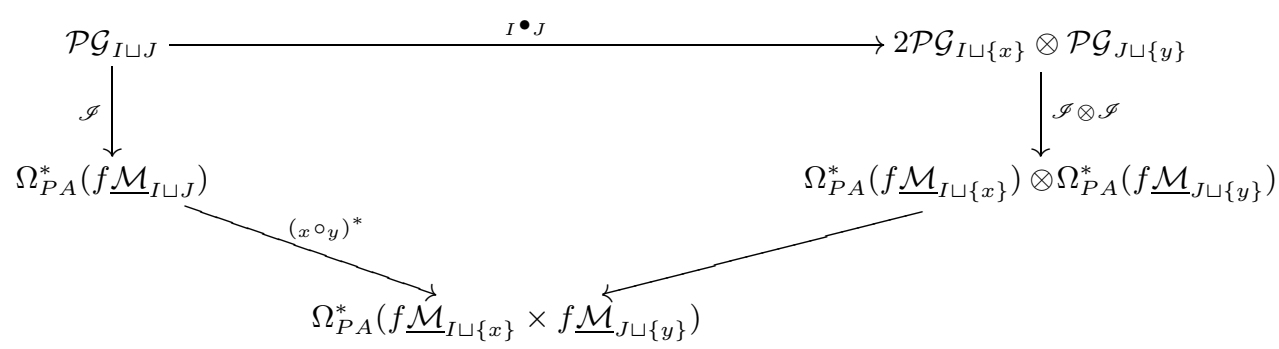

Proof. The composition map ${ }_{x}^{\circ}{ }_{y}: f \underline{\mathcal{M}}_{I \sqcup\{x\}} \times f \underline{\mathcal{M}}_{J \sqcup\{y\}} \rightarrow f \underline{\mathcal{M}}_{I \sqcup J}$ is a principal $S^{1}$-bundle over its image $\partial_{I, J}$, where the circle action is by rotating the rays at the $x$ and $y$ points in opposite directions. Consider a graph $\gamma \in \mathcal{P} \mathcal{G}_{I \sqcup J}$ with set of internal vertices $V_{\text {int }}$. There are pullback squares

$$
\begin{aligned}
& \coprod_{V_{\text {int }}=A \sqcup B} f \underline{\mathcal{M}}_{I \sqcup A \sqcup\{x\}} \times f \underline{\mathcal{M}}_{J \sqcup B \sqcup\{y\}} \stackrel{\amalg_{x^{\circ} y}}{\longrightarrow} \underbrace{}_{V_{\text {int }}=A \sqcup B} \partial_{I \sqcup A, J \sqcup B}
\end{aligned}
$$

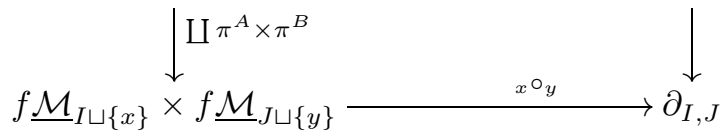

and

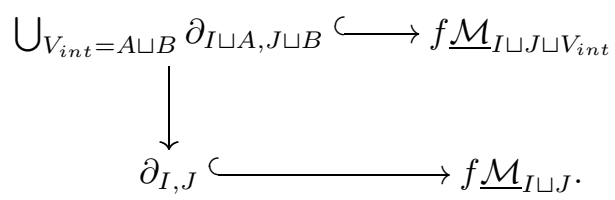

On each fibre over $\partial_{I, J}$ the quotient map $\amalg \partial_{I \sqcup A, J \sqcup B} \rightarrow \bigcup \partial_{I \sqcup A, J \sqcup B}$ is a diffeomorphism in the interior and finite-to- 1 on the boundary. With this observation and the above pullback squares, one has

$$
\begin{aligned}
\left({ }_{x} \circ y\right)^{*} \mathscr{I}(\gamma) & =\left({ }_{x} \circ y\right)^{*}\left(\frac{1}{2^{\left|V_{i n t}\right|}}\left(\pi^{V_{i n t}}\right)_{!} \mathscr{I}(\widetilde{\gamma})\right) \\
& =\frac{1}{2^{|A|+|B|}} \sum_{A \sqcup B=V_{i n t}}\left(\pi^{A}\right)_{!} \otimes\left(\pi^{B}\right)_{!}\left(\left({ }_{x} \circ_{y}\right)^{*} \mathscr{I}(\widetilde{\gamma})\right),
\end{aligned}
$$

where we choose compatible fibrewise orientations for $\pi^{A}$ and $\pi^{B}$. The lemma now follows directly from this, Lemma [7.1] and the definition of the cooperad structure on $\mathcal{P G}$ from section 4.4 
7.1. Proof of Theorem A. The projective graph complexes $\left\{\mathcal{P} \mathcal{G}_{V}\right\}$ collectively form a cyclic cooperad in the category of commutative DGAs, and they are degreewise finite dimensional. Hence the graded duals $\left\{\mathcal{P} \mathcal{G}_{V}^{\vee}\right\}$ form a cyclic operad in the category of cocommutative differential graded coalgebras. The projections $\mathcal{P G}_{V} \rightarrow H^{*}\left(f \underline{\mathcal{M}}_{V} ; \mathbb{R}\right)$ determine a quasi-isomorphism of cyclic cooperads, so the dual morphisms

$$
H_{*}\left(f \underline{\mathcal{M}}_{V} ; \mathbb{R}\right) \rightarrow \mathcal{P} \mathcal{G}_{V}^{\vee}
$$

constitute a quasi-isomorphism of cyclic operads. Let

$$
C_{*}^{S A}(-): \text { Semi-algebraic sets } \rightarrow \text { Chain complexes }
$$

denote the symmetric monoidal functor of semi-algebraic chains with real coefficients 11. This functor is weakly equivalent to the functor of real singular chains $C_{*}^{\text {sing }}(-)$, so the cyclic operads $C_{*}^{\text {sing }}(f \underline{\mathcal{M}} ; \mathbb{R})$ and $C_{*}^{S A}(f \underline{\mathcal{M}})$ are weakly equivalent. By [11, Prop. 7.3], if $X$ is compact, then the natural evaluation map

$$
C_{*}^{S A}(X) \rightarrow \Omega_{P A}^{*}(X)^{\vee}
$$

is a quasi-isomorphism. Although the complexes $\Omega_{P A}^{*}\left(f \underline{\mathcal{M}}_{V}\right)^{\vee}$ do not form a cyclic operad, the compositions

$$
C_{*}^{S A}\left(f \underline{\mathcal{M}}_{V}\right) \rightarrow \Omega_{P A}^{*}\left(f \underline{\mathcal{M}}_{V}\right)^{\vee} \stackrel{\mathscr{I}^{\vee}}{\longrightarrow} \mathcal{P} \mathcal{G}_{V}^{\vee}
$$

give a quasi-isomorphism of cyclic operads by Lemma 7.2. This completes the proof.

\section{REFERENCES}

[1] V. I. Arnold, The cohomology ring of the colored braid group. Mat. Zametki 5 (1969), 227-231. MR0242196 (39:3529)

[2] R. Budney, The operad of framed discs is cyclic. J. Pure Appl. Algebra 212 (2008), no. 1, 193-196. MR2355044 (2008k:55022)

[3] I. Gálvez-Carrillo, A. Tonks, and B. Vallette, Homotopy Batalin-Vilkovisky algebras. To appear in Journal of Noncommutative Geometry. arXiv:0907.2246.

[4] E. Getzler and J. Jones, Operads, homotopy algebra and iterated integrals for double loop spaces. arXiv:hep-th/9403055.

[5] E. Getzler, Batalin-Vilkovisky algebras and two-dimensional topological field theories. Comm. Math. Phys. 159 (1994), no. 2, 265-285. MR.1256989 (95h:81099)

[6] E. Getzler and M. M. Kapranov, Cyclic operads and cyclic homology. Geometry, topology, \& physics, 167-201, Conf. Proc. Lecture Notes Geom. Topology, IV, Int. Press, Cambridge, MA, 1995. MR1358617 (96m:19011)

[7] J. Giansiracusa and P. Salvatore, Formality of the framed little 2-discs and semidirect products. To appear in Proceedings of "Homotopy Theory of Function Spaces and Related Topics," Oberwolfach, April 5-11, 2009.

[8] J. Giansiracusa, The framed little 2-discs operad and diffeomorphisms of handlebodies. J. Topol. 4 (2011), no. 4, 919-941. MR.2860346

[9] F. Guillén Santos, V. Navarro, P. Pascual, and A. Roig, Moduli spaces and formal operads. Duke Math. J. 129 (2005), no. 2, 291-335. MR2165544 (2006e:14033)

[10] S. Halperin and J. Stasheff, Obstructions to homotopy equivalences. Adv. in Math. 32 (1979) n.3, 233-279. MR.539532 (80j:55016)

[11] R. Hardt, P. Lambrechts, V. Turchin and I. Volic, Real homotopy theory of semi-algebraic sets. arXiv:0806.0476

[12] W. J. Harvey, Boundary structure of the modular group. Riemann surfaces and related topics: Proceedings of the 1978 Stony Brook Conference (State Univ. New York, Stony Brook, N.Y., 1978), pp. 245-251, Ann. of Math. Stud., 97, Princeton Univ. Press, Princeton, N.J., 1981. MR624817 (83d:32022)

[13] S. Keel, Intersection theory of moduli space of stable $n$-pointed curves of genus zero. Trans. Amer. Math. Soc. 330 (1992), no. 2, 545-574. MR.1034665 (92f:14003) 
[14] T. Kimura, J. Stasheff, and A. Voronov, On operad structures of moduli spaces and string theory. Comm. Math. Phys. 171, 1-25 (1995). MR1341693 (96k:14019)

[15] M. Kontsevich, Operads and motives in deformation quantization. Lett. Math. Phys. 48 (1999) 35-72. MR1718044 (2000j:53119)

[16] M. Kontsevich, Deformation Quantization of Poisson Manifolds. Lett. Math. Phys. 66 (2003) 157-216. MR2062626(2005i:53122)

[17] P. Lambrechts and I. Volic. Formality of the little $N$-disks operad. arXiv:0808.0457

[18] P. Lambrechts and V. Turchin, Homotopy graph-complex for configuration and knot spaces. Trans. Amer. Math. Soc. 361 (2009), no. 1, 207-222. MR2439404(2009e:55016)

[19] P. Salvatore, Configuration spaces with summable labels. Cohomological methods in homotopy theory 375-395, Progr. Math., 196, Birkhäuser, Basel, 2001. MR1851264 (2002f:55039)

[20] P. Salvatore and N. Wahl, Framed discs operads and Batalin-Vilkovisky algebras, Q. J. Math. 54 (2003), 213-231. MR1989873 (2004e:55013)

[21] P. Severa, Formality of the chain operad of framed little disks. Lett. Math. Phys. 93 (2010), 29-35. MR 2661521

[22] P. Severa and T. Willwacher, Equivalence of formalities of the little discs operad. arXiv:0905.1789

[23] D. Sinha, Manifold-theoretic compactifications of configuration spaces. Selecta Math. (N.S.) 10 (2004), no. 3, 391-428. MR2099074 (2005h:55015)

[24] D. Tamarkin, Formality of chain operad of little discs. Lett. Math. Phys. 66 (2003), no. 1-2, 65-72. MR2064592(2005j:18010)

[25] D. Tamarkin, Another proof of M. Kontsevich formality theorem. arXiv:math/9803025

Department of Mathematics, Swansea University, Singleton Park, Swansea, SA2 8PP, UNITED Kingdom

E-mail address: j.h.giansiracusa@gmail.com

Dipartimento di Matematica, Universitá di Roma "Tor Vergata", Via della Ricerca SCIENTIFicA, 00133 Roma, Italy

E-mail address: salvator@mat.uniroma2.it 